\title{
MINIMIZERS OF THE LAWRENCE-DONIACH ENERGY IN THE SMALL-COUPLING LIMIT: FINITE WIDTH SAMPLES IN A PARALLEL FIELD
}

\author{
S. ALAMA ${ }^{\mathrm{a}, 1}$, A.J. BERLINSKY ${ }^{\mathrm{b}, 1}$, L. BRONSARD ${ }^{\mathrm{a}, 1}$ \\ ${ }^{a}$ Department of Mathematics and Statistics, McMaster Univ., Hamilton, Ontario, Canada L8S 4K1 \\ ${ }^{\mathrm{b}}$ Department of Physics and Astronomy, McMaster Univ., Hamilton, Ontario, Canada L8S 4K1
}

Received 8 November 2000

ABSTRACT. - In this paper we study the Lawrence-Doniach model for layered superconductors, for a sample with finite width subjected to a magnetic field parallel to the superconducting layers. We provide a rigorous analysis of the energy minimizers in the limit as the coupling between adjacent superconducting layers tends to zero. We identify a unique global minimizer of the Gibbs free energy in this regime ("vortex planes"), and reveal a sequence of first-order phase transitions by which Josephson vortices are nucleated via the boundary. The small coupling limit is studied via degenerate perturbation theory based on a Lyapunov-Schmidt decomposition which reduces the Lawrence-Doniach system to a finite-dimensional variational problem. Finally, a lower bound on the radius of validity of the perturbation expansion (in terms of various parameters appearing in the model) is obtained.

(C) 2002 L'Association Publications de l'Institut Henri Poincaré. Published by Elsevier B.V. All rights reserved

RÉSUMÉ. - Nous analysons rigoureusement les minimiseurs du modèle de Lawrence-Doniach pour les supraconducteurs en couche quand le paramètre de couplage de Josephson tend vers zero. Le champs magnétique imposé est parallèle aux plans supraconductifs et l'échantillon est de largeur fini. A l'aide d'une méthode de perturbation dégénérée basée sur une décomposition de Lyapunov-Schmidt, nous réduisons le système de Lawrence-Doniach à un problème variationel de dimension fini. Nous obtenons un minimiseur global unique ("les plans de vortex") et montrons qu'il y a nucléations des vortex de Josephson à la frontière via une suite de transitions de premier ordre. Finalement, à l'aide d'estimations a-priori nous obtenons aussi une borne inférieure sur le rayon de convergence du développement de Taylor en termes de paramètres présents dans le modèle.

(C) 2002 L'Association Publications de l'Institut Henri Poincaré. Published by Elsevier B.V. All rights reserved

\section{Introduction}

In 1971 Lawrence and Doniach [18] introduced a Ginzburg-Landau type model for superconducting materials with a planar layered structure. In this model, the

\footnotetext{
${ }^{1}$ Supported by an NSERC Research Grant.
} 
superconductor occupies an array of parallel sheets with insulating material acting as a buffer between the sheets. While this model was originally proposed to study layered structures artificially produced by successively deposing thin planar sheets of superconducting metal with organic separators, it has received renewed attention due to the discovery of high temperature superconductors. Indeed, nearly all of these high$T_{c}$ materials are crystals with a distinctly layered structure, consisting of copper oxide superconducting planes stacked with intervening insulating (or weakly superconducting) planes.

In this paper we will consider the case of a layered superconductor in a uniform magnetic field imposed parallel to the superconducting planes. We assume that there are a finite number of superconducting sheets, each parallel to the $x y$-plane, with uniform separation $p$. We assume that the external magnetic field is applied along the $y$-direction, $\vec{H}=H \hat{y}$. We will take the planes to be of infinite extent in the $y$-direction, and assume the local magnetic field will be everywhere independent of $y$ and point in the $y$-direction,

$$
\vec{h}(x, y, z)=h(x, z) \hat{y} .
$$

The vector potential $\vec{A}$ may then be chosen to lie in the $x z$-plane,

$$
\vec{A}(x, y, z)=A_{x}(x, z) \hat{x}+A_{z}(x, z) \hat{z}, \quad \vec{h}=\operatorname{curl} \vec{A}=\left(\frac{\partial A_{x}}{\partial z}-\frac{\partial A_{z}}{\partial x}\right) \hat{y} .
$$

We assume that the sample has fixed width $2 L$ in the $x$-direction, and hence the superconducting sheets are described by the stack of parallel planar strips

$$
\Sigma_{N}:-L \leqslant x \leqslant L, \quad-\infty<y<\infty, \quad z=z_{n}:=n p, n=0,1, \ldots, N .
$$

Since each sheet $\Sigma_{N}$ is superconducting it carries a (complex-valued) order parameter $\psi_{n}(x), n=0, \ldots, N$. We choose units in such a way that $\left|\psi_{n}\right|=1$ represents a purely superconducting state. The Lawrence-Doniach model is then formulated in terms of the following Gibbs free energy functional:

$$
\begin{aligned}
\mathcal{G}_{r}\left(\psi_{n}, \vec{A}\right)= & \frac{H_{c}^{2}}{4 \pi}\left\{p \sum_{n=0}^{N} \int_{-L}^{L}\left[\frac{1}{\kappa^{2}}\left|\left(\frac{\mathrm{d}}{\mathrm{d} x}-\mathrm{i} A_{x}\right) \psi_{n}\right|^{2}+\frac{1}{2}\left(\left|\psi_{n}\right|^{2}-1\right)^{2}\right] \mathrm{d} x\right. \\
& +\frac{r}{2} p \sum_{n=1}^{N} \int_{-L}^{L}\left|\psi_{n}-\psi_{n-1} \exp \left(\mathrm{i} \int_{z_{n-1}}^{z_{n}} A_{z}(x, s) \mathrm{d} s\right)\right|^{2} \mathrm{~d} x \\
& \left.+\frac{1}{\kappa^{2}} \int_{-L}^{L} \int_{0}^{N p}\left(\frac{\partial A_{x}}{\partial z}-\frac{\partial A_{z}}{\partial x}-H\right)^{2} \mathrm{~d} z \mathrm{~d} x\right\},
\end{aligned}
$$

where $r$ is the interlayer coupling parameter (or Josephson coupling parameter). We have chosen units such that the in-plane penetration depth $\lambda_{a b}=1, \kappa=\lambda_{a b} / \xi_{a b}$ is the Ginzburg-Landau parameter $\left(\lambda_{a b}, \xi_{a b}\right.$ are the in-plane penetration depth and correlation length, respectively), and the magnetic fields are measured in units of $H_{c} / \kappa$, where $H_{c}$ is the thermodynamic critical field. (See [24].) 
The coupling between the superconducting planes given by the second sum in $\mathcal{G}_{r}$ simulates the Josephson effect, by which superconducting electrons travel from one superconducting sheet to another by quantum mechanical tunnelling. We will see this explicitly in the Euler-Lagrange equations, where the currents in the gaps between planes will be determined by the sine of the gauge-invariant phase difference. The interlayer coupling parameter $r$ gives the strength of the Josephson effect. In our units,

$$
r=\frac{2}{\lambda_{J}^{2} \kappa^{2} p^{2}}
$$

where $\lambda_{J}$ is the (non-dimensional) Josephson penetration depth. In an anisotropic Ginzburg-Landau model, $\lambda_{J}^{2}$ gives the effective mass ratio which determines the degree of anisotropy. For highly anisotropic superconductors $\lambda_{J}$ is very large, and hence when $\kappa p \sim 1$ we may treat $r$ as a small parameter.

Due to the layered structure one expects these materials to be highly anisotropic. A first attempt to model layered superconductors is by an anisotropic Ginzburg-Landau model, which treats the sample as a three-dimensional solid with anisotropic material parameters. For certain materials and temperatures close to the critical temperature $T_{c}$ this approximation seems valid, but for the most anisotropic superconductors the anisotropic Ginzburg-Landau model does not give a good qualitative or quantitative description of experimental observations. For example, when the sample is subjected to a sufficiently strong magnetic field oriented parallel to the superconducting planes Kes, Aarts, Vinokur, and van der Beek [16] observe a transition betwen "three-dimensional" behavior (governed by the anisotropic Ginzburg-Landau model) and "two-dimensional" behavior at a critical temperature $T_{C 0}$ below $T_{C}$. In the two-dimensional regime the superconducting planes decouple and the applied magnetic field penetrates completely between the planes, virtually unscreened by the superconductor. Despite the penetration of the field, superconductivity within the planes is not destroyed even in very strong applied fields. This "magnetically transparent" state is inconsistent with the anisotropic Ginzburg-Landau model, where magnetic fields of moderate intensity are largely expelled from the bulk except for an array of isolated vortices (the "Abrikosov lattice"). In addition the Ginzburg-Landau model predicts the breakdown of superconductivity when the applied field penetrates the material completely, which occurs when the field exceeds a critical value $H_{c 2}$.

We note that Chapman, Du and Gunzburger [9] have proven that solutions of the Lawrence-Doniach model converge to solutions of the anisotropic Ginzburg-Landau model (and in particular the convergence of energy minimizers) under the limit $p \rightarrow 0$ with $\kappa, \lambda_{J}$ fixed. This limit does not correspond to our "two-dimensional" regime, since it would send $r \rightarrow \infty$, corresponding to a strong coupling between adjacent superconducting layers. Indeed, we observe that the non-dimensional separation distance $p$ of our model is related to the (dimensionally dependent) physical separation $\bar{p}$ via $p=\bar{p} / \lambda_{a b}$. Since $\lambda_{a b}(T) \rightarrow \infty$ as $T \rightarrow T_{c}$, the limit $T \rightarrow T_{c}$ effectively corresponds to $p \rightarrow 0$ (with $\kappa, \lambda_{J}, \bar{p}$ fixed) in our units, and therefore the Chapman, Du and Gunzburger limit can be interpreted as letting $T \rightarrow T_{c}$. This is consistent with the observed "dimensional crossover" to the anisotropic Ginzburg-Landau regime for temperatures near $T_{c}$. 
In this paper we will study the minimizers (and low-energy solutions) of the Lawrence-Doniach system for $r$ near zero and thereby analyse the structure of the resulting "transparent state". The crucial observation is that when $r=0$ the planes decouple, and the energy may be minimized explicitly by solving simple first order equations. Even after gauge symmetries have been removed the $r=0$ problem exhibits an additional symmetry, corresponding to an $N$-dimensional torus action (where $N+1$ denotes the number of superconducting planes), and thus the minimization problem at $r=0$ degenerates on a finite dimensional manifold in function space. We can think of the $r=0$ problem in analogy with the self-dual point of the Ginzburg-Landau model $(\kappa=1 / \sqrt{2}$ ), where minimizers satisfy a first-order Bogomolnyi system (in addition to the usual second order Ginzburg-Landau equations), and the same minimum energy is obtained by any configuration of vortices.

When $r \neq 0$ this symmetry is broken and a Lyapunov-Schmidt decomposition reduces the problem of finding solutions with $r \simeq 0$ to a finite dimensional variational problem on the degenerate manifold. The minimum value of energy is $\mathrm{O}(r)$, and we indeed recover the "transparent state" observed in experiments. The local magnetic field $h(x, z)=H+\mathrm{O}(r)$ inside the sample, and superconductivity is hardly affected in each plane, $\left|\psi_{n}(x)\right|=1-\mathrm{O}(r)$. In particular the order parameters are never zero: "vortices" correspond to local maxima of the local magnetic field, and lie between the layers. In the physics literature these are referred to as Josephson vortices, as opposed to the Abrikosov vortices typically observed in the Ginzburg-Landau theory.

The finite dimensional reduced problem may be solved explicitly to determine the exact geometry of the Josephson vortex lattice. For a finite sample in $x, z$ the minima of energy form "vortex plane" configurations, in which the local magnetic field is uniform in $z$. The vortices are not separated, but line up vertically at the local maxima of $h(x, z)=h(x)$. (See Fig. 1.) As the external field $H$ is increased vortices are nucleated at the edges, by a first-order phase transition. Formal asymptotic expansions for these solutions were calculated by Theorodakis [23], and Kuplevakhsky [17] claimed that they were the only solutions of the Lawrence-Doniach system. On the contrary, we find that there are exactly $2^{N}$ solutions with energy $\mathrm{O}(r)$ : two represent vortex planes (one stable and the other unstable), and the others (unstable) lattices of various geometries. (See Theorem 4.1.)

Again, we note the distinction with the Ginzburg-Landau model: the geometry of the Abrikosov lattice was determined by numerical comparison of a finite number of possible lattice geometries. For the Lawrence-Doniach model in the small coupling limit we are able to identify the absolute minimizer (and all low-energy solutions of the Euler-Lagrange equations) explicitly and rigorously. This is a direct benefit of the discrete nature of the model.

The basic idea that an infinite dimensional variational problem is actually governed by a finite dimensional one in some parameter limit is a common one in analysis. Indeed, it appears in such diverse contexts as the location of Ginzburg-Landau vortices as $\kappa \rightarrow \infty$ (Bethuel, Brezis and Hélein [5], Bethuel and Riviére [6]), spike-layer solutions (for example, Li and Nirenberg [19], Gui [14], or Wei [25]), and blow-up for critical exponent problems (for example in Bahri, Li and Rey [4], or Rey [22]). These examples are of singular perturbation problems, though. The transparent state arises as a degenerate 


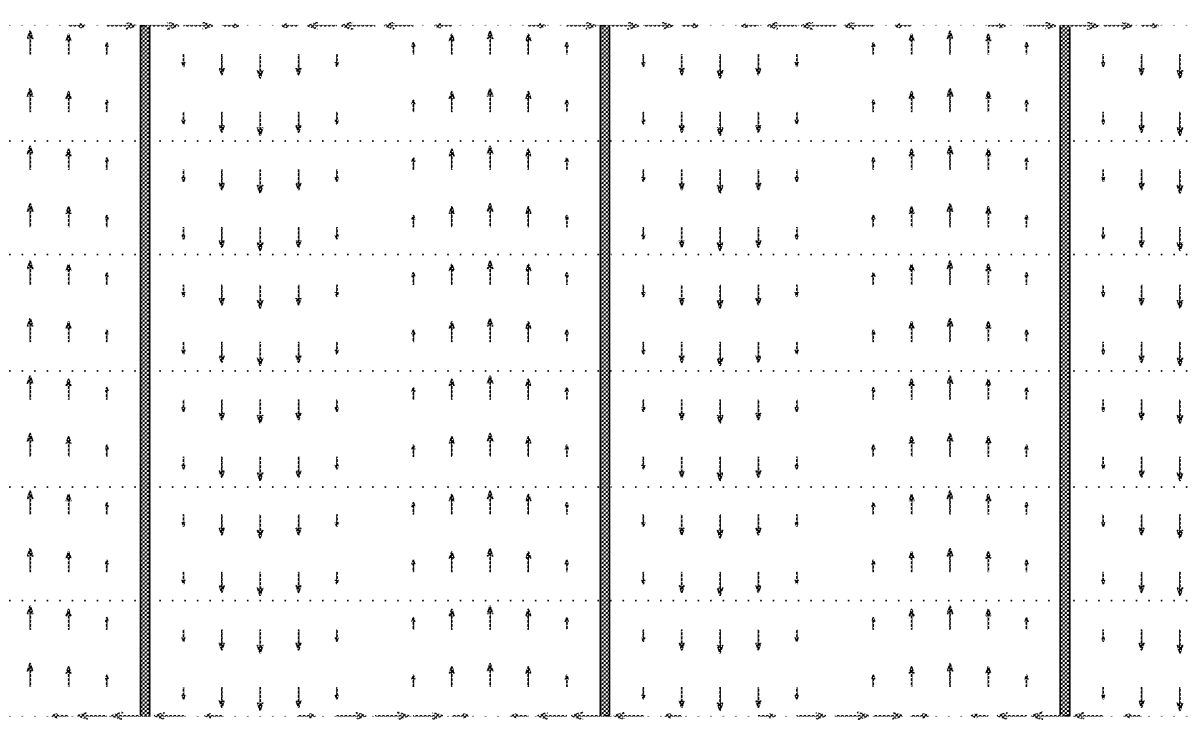

Fig. 1. Vortex planes, for a sample with a finite number of superconducting planes. The dotted horizontal lines represent the superconducting planes, and horizontal arrows indicate the in-plane currents $j_{x}^{(n)}$, which vanish to order $r$ in the interior of the sample. The vertical arrows indicate the Josephson currents $j_{z}^{(n)}$ in each gap. Except for an edge effect near the top and bottom of the sample, the magnetic field $h(x, z)$ and Josephson currents $j_{z}$ are $z$-independent. The vortices correspond to local maxima of $h$, and lie along planes $x=$ constant, indicated here by the dark bands.

regular perturbation of the $r=0$ problem, and hence it is more closely related to the work of Ambrosetti, Coti-Zelati and Ekeland [3] and Ambrosetti and Badiale [2] on homoclinic solutions of Hamiltonian systems and the Poincaré-Melnikov functions.

Analytically our results are unambiguous: for any choice of the other parameters $(L, \kappa, N, H, p)$ we can choose $r$ sufficiently small so that the vortex planes configuration minimizes the free energy. However, in a real superconductor $r$ is not infinitessimally small, which raises the question of how the interval of validity of the $r$-expansion is affected by the values of the other parameters in the problem. For example, in the order $r$ term in the expansion of the solution (see (34), for example) we observe "secular" terms, that is factors which become large without bound as the length of the interval increases, and in general remark that the coefficients increase with $L, \kappa$ and decrease with $H$. We address this question in Section 5 , where we produce a lower bound for the interval of validity as a function of $N, L, \kappa$, and $H$. We discover that this interval is independent of $N$ and indeed increases with decreasing $L, \kappa$ and increasing $H$. For the high- $T_{c}$ superconductors, $\kappa$ is large and typical macroscopic sample widths $L$ are very large compared with the in-plane penetration depth $\lambda_{a b}$. This indicates that our analysis may be more applicable to experiments with highly anisotropic organic or synthetic multi-layer superconductors, where the material parameters are significantly different from the high- $T_{c}$ crystals.

In a subsequent paper [1] we address the question of minimizers of the LawrenceDoniach energy in very large samples, by considering periodic solutions in an infinitely 
wide sample. In fact, in the periodic case we find a different geometry for the energy minimizing configuration! The periodic solution with the least energy is a lattice with period two in $n$, forming a diamond pattern of Josephson vortices, proposed by Bulaevskiı and Clem [8]. In the periodic case, the role of $L$ is now taken by the period, which for minimizers will decrease as the applied field $H$ increases. Therefore the small $r$ expansion will have a large range of validity in sufficiently large fields $H$, and the result should better describe experiments with high- $T_{c}$ superconductors.

\subsection{Variational setting}

We begin with the following basic energy estimate, which legitimizes the simplification $\left|\psi_{n}(x)\right| \simeq 1$ for small $r$. Indeed, in the physics literature the approximation $\left|\psi_{n}\right| \sim 1$ is widely assumed to hold: see for example Bulaevskii [7], Clem and Coffey [10], or Bulaevskii and Clem [8]. In particular, we see immediately that in the regime $r \simeq 0$ there will be no "Abrikosov vortices" in the sense that the order parameter is never zero.

Proposition 1.1. - Let $D:=[-L, L] \times[0, N p]$. For any $r \geqslant 0$ we have

$$
\inf \left\{\frac{4 \pi}{H_{c}^{2}} \mathcal{G}_{r}\left(\psi_{n}, \vec{A}\right): \psi_{n} \in H^{1}([-L, L]), \vec{A} \in H^{1}\left(D ; \mathbf{R}^{2}\right)\right\} \leqslant 2 N p\left(L+\frac{1}{p H}\right) r .
$$

Moreover, there exist constants $r_{0}>0$ and $C=C(N, L, \kappa, H, p)>0$ such that for all $r \in\left[0, r_{0}\right)$ the minimum is attained by $\left(\psi_{n}, \vec{A}\right)$ with $\left|\psi_{n}(x)\right| \geqslant 1-C r^{1 / 2}>0$ for all $x \in[-L, L]$.

Proof. - We choose a test configuration, $\psi_{n}(x)=\exp \{\operatorname{inp} H x\}, A=(H z, 0)$ to estimate the free energy,

$$
\frac{4 \pi}{H_{c}^{2}} \mathcal{G}_{r}\left(\psi_{n}, A\right)=r p \sum_{n=1}^{N} \int_{-L}^{L}(1-\cos (p H x)) \mathrm{d} x \leqslant 2 N\left(L+\frac{1}{p H}\right) p r .
$$

The fact that the minimum is attained can be easily proven once an appropriate choice of gauge has been made: see [9] for details.

It remains to show that the order parameters lie near the unit circle. We recall the "Diamagnetic inequality" (see for example p. 174 of [20]), which states

$$
|\nabla| f||(x) \leqslant|(\nabla-\mathrm{i} A) f|(x) \quad \text { (almost everywhere) }
$$

for every $f \in L_{l o c}^{2}$ with $(\nabla-\mathrm{i} A) f \in L_{l o c}^{2}$. Using the elementary inequality $\left(1-\left|\psi_{n}\right|\right)^{2} \leqslant$ $\left(1-\left|\psi_{n}\right|^{2}\right)^{2}$, and the energy bound we obtain

$$
\begin{aligned}
\sum_{n=0}^{N}\left\|1-\left|\psi_{n}\right|\right\|_{H^{1}}^{2} & \leqslant \sum_{n=0}^{N} \int_{-L}^{L}\left(\left(1-\left|\psi_{n}\right|^{2}\right)^{2}+\frac{1}{\kappa^{2}}\left|\frac{\mathrm{d}}{\mathrm{d} x}\right| \psi_{n}||^{2}\right) \mathrm{d} x \\
& \leqslant \sum_{n=0}^{N} \int_{-L}^{L}\left(\left(1-\left|\psi_{n}\right|^{2}\right)^{2}+\frac{1}{\kappa^{2}}\left|\left(\frac{\mathrm{d}}{\mathrm{d} x}-\mathrm{i} A_{x}\right) \psi_{n}\right|^{2}\right) \mathrm{d} x
\end{aligned}
$$




$$
\leqslant \frac{4 \pi}{H_{c}^{2}} \mathcal{G}_{r}\left(\psi_{n}, A\right) \leqslant 2 N\left(L+\frac{1}{H p}\right) p r .
$$

Here we choose an equivalent norm for $H^{1}([-L, L])$,

$$
\|f\|_{H^{1}}:=\sqrt{\int_{-L}^{L}\left(|f|^{2}+\frac{1}{\kappa^{2}}\left|f^{\prime}\right|^{2}\right) \mathrm{d} x} .
$$

By the Sobolev embedding we have for each $n=0, \ldots, N$ that $\left|\psi_{n}(x)\right| \geqslant 1-C r^{1 / 2}$ with constant $C$ depending on $N, L, p, \kappa, H$.

The above proposition suggests the use of polar coordinates for $\psi_{n}$ in order to deal more directly with the phase of the order parameter, which plays the essential role in Josephson coupling. We define $f_{n}, \phi_{n}$ via $\psi_{n}(x)=f_{n}(x) \exp \left(\mathrm{i} \phi_{n}(x)\right)$, and note that $\phi_{n}$ is well-defined only up to an additive integer multiple of $2 \pi$. We then define our new free energy in terms of the variables $\left(f_{n}, \phi_{n}, \vec{A}\right)$ to coincide with $\frac{4 \pi}{H_{c}^{2}} \mathcal{G}_{r}$, that is:

$$
\begin{aligned}
\Omega_{r}\left(f_{n}, \phi_{n}, \vec{A}\right)= & p \sum_{n=0}^{N} \int_{-L}^{L}\left[\frac{1}{2}\left(f_{n}^{2}-1\right)^{2}+\frac{1}{\kappa^{2}}\left(f_{n}^{\prime}\right)^{2}+\frac{1}{\kappa^{2}}\left(\phi_{n}^{\prime}-A_{x}\left(x, z_{n}\right)\right)^{2} f_{n}^{2}\right] \mathrm{d} x \\
& +\frac{r}{2} p \sum_{n=1}^{N} \int_{-L}^{L}\left(f_{n}^{2}+f_{n-1}^{2}-2 f_{n} f_{n-1} \cos \left(\Phi_{n, n-1}\right)\right) \mathrm{d} x \\
& +\frac{1}{\kappa^{2}} \int_{-L}^{L} \int_{0}^{N p}\left(\frac{\partial A_{x}}{\partial z}-\frac{\partial A_{z}}{\partial x}-H\right)^{2} \mathrm{~d} z \mathrm{~d} x,
\end{aligned}
$$

where

$$
\Phi_{n, n-1}(x):=\phi_{n}-\phi_{n-1}-\int_{z_{n-1}}^{z_{n}} A_{z}(x, z) \mathrm{d} z
$$

is the gauge-invariant phase difference, $z_{n}=n p$ and $r=\frac{2}{\lambda_{J}^{2} \kappa^{2} p^{2}}$. When $f_{n}=\left|\psi_{n}\right|$ is bounded away from zero the condition $\psi_{n} \in H^{1}([-L, L])$ is equivalent to both $f_{n}, \phi_{n} \in H^{1}([-L, L])$.

We first define a base space, in which $\Omega_{r}$ will be a smooth functional:

$$
\mathcal{E}:=\left\{\begin{array}{cl}
\left(f_{n}, \phi_{n}, \vec{A}\right): & f_{n} \in H^{1}([-L, L]), \phi_{n} \in H^{1}([-L, L]), n=0, \ldots, N \\
& \vec{A}=\left(A_{x}, A_{z}\right) \in H^{1}\left(D, \mathbf{R}^{2}\right)
\end{array}\right\} .
$$

We remark that we should really work in the convex subset of $\mathcal{E}$ with $f_{n} \geqslant 0$ for all $n=0, \ldots, N$, but Lemma 1.1 already guarantees that the solutions we will find will have $f_{n} \sim 1$. Furthermore, Proposition 4.5 of [9] asserts that $f_{n}(x)=\left|\psi_{n}(x)\right| \leqslant 1$ for any solution of the Lawrence-Doniach equations. 
We note that $\Omega_{r}$ is a smooth $\left(C^{\infty}\right)$ functional on $\mathcal{E}$, and that variation with respect to each of its arguments gives the Lawrence-Doniach system. First, we denote by

$$
V_{n}:=\left(\phi_{n}^{\prime}-A_{x}\left(x, z_{n}\right)\right),
$$

the supercurrent velocity. Then, variation of $\Omega_{r}$ with respect to $f_{n}$ (for each $n=$ $0, \ldots, N)$ yields

$$
\begin{array}{ll}
- & \frac{1}{\kappa^{2}} f_{n}^{\prime \prime}+\left(f_{n}^{2}-1\right) f_{n}+\frac{1}{\kappa^{2}} V_{n}^{2} f_{n} \\
& = \begin{cases}\frac{r}{2}\left(f_{n-1} \cos \Phi_{n, n-1}+f_{n+1} \cos \Phi_{n+1, n}-2 f_{n}\right), & n \neq 0, N ; \\
\frac{r}{2}\left(f_{1} \cos \Phi_{1,0}-f_{0}\right), & n=0 ; \\
\frac{r}{2}\left(f_{N-1} \cos \Phi_{N, N-1}-f_{N}\right), & n=N,\end{cases}
\end{array}
$$

with boundary condition $f_{n}^{\prime}( \pm L)=0, n=0, \ldots, N$.

Variation with respect to $A_{x}$ produces the following equation in weak form:

$$
\begin{aligned}
& \frac{\partial h}{\partial z}(x, z)=0 \quad \text { for all }(x, z) \in(-L, L) \times\left(z_{n-1} z_{n}\right), n=0, \ldots, N, \\
& h\left(x, z_{n}+\right)-h\left(x, z_{n}-\right)=-p f_{n}^{2}(x)\left(\phi_{n}^{\prime}-A_{x}\left(x, z_{n}\right)\right), \quad n=1, \ldots, N-1, \\
& h\left(x, z_{N}-\right)=p f_{N}^{2}(x)\left(\phi_{N}^{\prime}-A_{x}\left(x, z_{N}\right)\right), \quad h(x, 0+)=-p f_{0}^{2}(x)\left(\phi_{0}^{\prime}-A_{x}(x, 0)\right) .
\end{aligned}
$$

In other words, $h$ is independent of $z$ away from the superconducting planes, and supercurrents in the SC planes create jump discontinuities. The dependence of $h(x, z)$ on $x$ in each gap is determined by (compactly supported) variations of $A_{z}$ :

$$
\begin{aligned}
& \frac{\partial h}{\partial x}=\frac{r \kappa^{2} p}{2} f_{n}(x) f_{n-1}(x) \sin \Phi_{n, n-1}(x), \\
& \quad \text { if }-L \leqslant x \leqslant L \text { and } z_{n-1}<z<z_{n}, n=1, \ldots, N ;
\end{aligned}
$$

with boundary condition $h( \pm L, z)=H$. It is therefore natural to define

$$
h(x, z)=h^{(n)}(x), \quad \text { when }-L \leqslant x \leqslant L, z_{n-1}<z<z_{n}, n=1, \ldots, N,
$$

with $h^{(n)}$ determined by the ordinary differential equations (5) together with the boundary condition $h^{(n)}( \pm L)=H$.

Variation with respect to $\phi_{n}$ produces the following current-conservation laws:

$$
\begin{array}{ll}
\frac{1}{\kappa^{2}} \frac{\mathrm{d}}{\mathrm{d} x}\left(f_{n}^{2}\left(\phi_{n}^{\prime}-A_{x}\left(x, z_{n}\right)\right)\right) \\
\quad= \begin{cases}\frac{r}{2}\left[f_{n} f_{n-1} \sin \Phi_{n, n-1}-f_{n+1} f_{n} \sin \Phi_{n+1, n}\right], & n=1, \ldots, N-1 ; \\
-\frac{r}{2} f_{1} f_{0} \sin \Phi_{1,0}, & n=0 ; \\
\frac{r}{2} f_{N} f_{N-1} \sin \Phi_{N, N-1}, & n=N,\end{cases}
\end{array}
$$

with boundary condition

$$
f_{n}^{2}(x)\left(\phi_{n}^{\prime}(x)-A_{x}\left(x, z_{n}\right)\right)=0, \quad x= \pm L, n=0, \ldots, N,
$$

which expresses the physical fact that current should not flow past the edge of the material. Note that (7) does not yield any new information, since it can be obtained by 
differentiating the jump condition (4) and substituting from (5). This is not surprising, since gauge invariance implies a nontrivial relationship between the $\phi_{n}$ and $\vec{A}$. Indeed, denoting the supercurrents in the planes by

$$
j_{x}^{(n)}:=V_{n} f_{n}^{2}
$$

and the Josephson current in between the $n-1$ and the $n$th planes by

$$
j_{z}^{(n)}:=\frac{r}{2} \kappa^{2} p f_{n} f_{n-1} \sin \Phi_{n, n-1},
$$

Eq. (7) is a semi-discrete version of the classical continuity equation $\operatorname{div} \vec{j}=0$, and gives the conservation law corresponding to the $U(1)$ gauge invariance in accordance with Noether's Theorem.

We are most interested in the gauge invariant "observable" quantities, which enter directly into the free energy: the density of superconducting eletrons $f_{n}$, the supercurrent velocity $V_{n}$, the gauge-invariant phase difference $\Phi_{n, n-1}$, and the local magnetic field $h(x, z)$. A very useful formula for $\Phi_{n, n-1}(x)$ can be obtained by applying Stokes' Theorem in the rectangle $R=(0, x) \times\left(z_{n-1}, z_{n}\right)$ :

$$
\Phi_{n, n-1}(x)=\int_{0}^{x}\left(V_{n}-V_{n-1}\right) \mathrm{d} \bar{x}+p \int_{0}^{x} h^{(n)}(\bar{x}) \mathrm{d} \bar{x}+\Phi_{n, n-1}(0), \quad n=1, \ldots, N .
$$

From these equations we easily verify the smoothness of observables associated to weak solutions of the Lawrence-Doniach system:

Proposition 1.2. - Suppose $\left(f_{n}, \phi_{n}, \vec{A}\right) \in \mathcal{E}$ are critical points of $\Omega_{r}$. Then $f_{n}, V_{n}, \Phi_{n, n-1} \in C^{\infty}([-L, L])$, and $h \in C^{\infty}\left([-L, L] \times\left(z_{n-1}, z_{n}\right)\right), n=1, \ldots, N$.

Of course, the regularity of the non-gauge-invariant quantities $\phi_{n}$ and $\vec{A}$ depends on the choice of gauge.

Remark 1.3. - Some authors have (correctly) pointed out that it is not physically correct to impose the external field via a Dirichlet condition $h=H$ on the boundary $\partial D$ of the sample. A more appropriate model for the effect of an external field is obtained by placing the superconductor $D$ in a larger region $\tilde{D} \supset D$, (with $\tilde{D}=\mathbf{R}^{2}$ possibly) and including the field energy in $\tilde{D} \backslash D$ in the calculations of the free energy,

$$
\tilde{\Omega}_{r}\left(f_{n}, \phi_{n}, \vec{A}\right):=\Omega_{r}\left(f_{n}, \phi_{n}, \vec{A}\right)+\iint_{\tilde{D} \backslash D}(\operatorname{curl} \vec{A}-H)^{2} \mathrm{~d} x \mathrm{~d} z .
$$

For example, our sample $D$ may be lying at the center of a long cylindrical solenoid of large radius (whose interior cross-section is a large disk $\tilde{D}$ ). All of the preceding analysis can then be carried through for $\tilde{\Omega}_{r}$ by choosing an appropriate space (see Rubinstein and Schatzman [21] for a discussion of the correct setting when $\tilde{D}=\mathbf{R}^{2}$ ), but it is easy to verify that the Euler-Lagrange equations yield $h(x, z) \equiv H$ for $(x, z) \in \tilde{D} \backslash D$. This is due to the two-dimensional ansatz: $\nabla \times(g(x, z) \hat{y})=0$ in a domain implies that $g$ is 
constant there. Consequently we may use the simpler form of the energy $\Omega_{r}$ with no loss of generality or of physical relevance.

There is a large degree of degeneracy of $\Omega_{r}$ in $\mathcal{E}$ due to the gauge invariance: if $\chi \in H^{2}([-L, L] \times[0, N p])$, and

$$
\hat{f}_{n}=f_{n}, \quad \hat{\phi}_{n}(x)=\phi_{n}(x)-\chi\left(x, z_{n}\right), \quad \hat{A}=\vec{A}-\nabla \chi,
$$

then $\Omega_{r}\left(\hat{f}_{n}, \hat{\phi}_{n}, \hat{A}\right)=\Omega_{r}\left(f_{n}, \phi_{n}, \vec{A}\right)$. As usual, we eliminate this troublesome degeneracy by fixing a gauge. The most convenient choice is the Coulomb gauge, which allows us to control the $H^{1}$ norm of the vector potential $\vec{A}$ by its curl. We define a subspace of $\mathcal{E}$ to incorporate this choice of gauge,

$$
\mathcal{E}_{g}:=\left\{\left(f_{n}, \phi_{n}, \vec{A}\right) \in \mathcal{E}: \int_{-L}^{L} \phi_{0}(x) \mathrm{d} x=0, \operatorname{div} \vec{A}=0 \text { in } D, \text { and } \vec{A} \cdot \vec{n}=0 \text { on } \partial D\right\} .
$$

This choice is made with no loss of generality:

Lemma 1.4. - For every $\left(f_{n}, \phi_{n}, \vec{A}\right) \in \mathcal{E}$, there exists $\chi \in H^{2}(D)$ so that $\left(f_{n}\right.$, $\left.\phi_{n}-\chi\left(\cdot, z_{n}\right), \vec{A}-\nabla \chi\right) \in \mathcal{E}_{g}$.

We also have:

LEMMA 1.5. - For every $\left(f_{n}, \phi_{n}, \vec{A}\right) \in \mathcal{E}_{g}$

$$
\|\vec{A}\|_{H^{1}(D)}^{2} \leqslant C_{0}\|\operatorname{curl} \vec{A}\|_{L^{2}(D)}^{2},
$$

where

$$
C_{0}=2\left[1+\frac{4}{\pi^{2}} \frac{L^{2} N^{2} p^{2}}{N^{2} p^{2}+4 L^{2}}\right]^{2} .
$$

Proof of Lemma 1.4. - Given $\left(f_{n}, \phi_{n}, \vec{A}\right) \in \mathcal{E}$ we may solve the linear Neumann problem,

$$
\begin{cases}\Delta \chi=\operatorname{div} \vec{A} & \text { for } x \in D, \\ \partial \chi / \partial \vec{n}=\vec{A} \cdot \vec{n} & \text { for } x \in \partial D .\end{cases}
$$

By the divergence theorem there exists a unique (up to constants) weak solution, $\chi \in H^{1}(D)$. Since $\vec{A} \in H^{1}$ we have $\chi \in H_{\text {loc }}^{2}(D)$ by standard regularity theory. Since the domain $D$ is polygonal we must be more careful to determine the regularity at the corners, but by Theorem 1.5.2.4 of Grisvard [13] we may find an $H^{2}(D)$ function whose normal derivative coincides with $\vec{A} \cdot \vec{n}$ on $\partial D$. Then Theorem 4.3.1.4 of [13] provides global regularity, $\chi \in H^{2}(D)$. By subtracting a constant from $\chi$ such that $\phi_{0}(x)-\chi(x, 0)$ has average zero we achieve the desired gauge change.

Proof of Lemma 1.5. - Assume $\left(f_{n}, \phi_{n}, \vec{A}\right) \in \mathcal{E}_{g}$. We now solve the Dirichlet problem,

$$
\begin{cases}\Delta \eta=\operatorname{curl} \vec{A} & \text { for } x \in D \\ \eta=0 & \text { for } x \in \partial D\end{cases}
$$


By Theorem 4.3.1.4 of [13] the unique solution $\eta \in H^{2}(D)$, and $\tilde{A}=\left(\partial_{z} \eta,-\partial_{x} \eta\right) \in$ $H^{1}\left(D ; \mathbf{R}^{2}\right)$ with $\operatorname{div}(\vec{A}-\tilde{A})=0, \operatorname{curl}(\vec{A}-\tilde{A})=0$, and $(\vec{A}-\tilde{A}) \cdot \vec{n}=0$ on $\partial D$. Hence $\vec{A}=\tilde{A}$, and the explicit solution of the Dirichlet problem in a rectangle (via Fourier analysis) provides the constant $C_{0}>0$ such that:

$$
\|\vec{A}\|_{H^{1}(D)}^{2} \leqslant\|\eta\|_{H^{2}(D)}^{2} \leqslant C_{0}\|\Delta \eta\|_{L^{2}(D)}^{2}=C_{0}\|\operatorname{curl} \vec{A}\|_{L^{2}(D)}^{2} .
$$

\section{Minimization at $r=0$}

When $r=0$, the superconducting planes decouple, and we may solve the minimization problem explicitly. The solution of the problem $r \sim 0$ will require some detailed second-order information on the minimizers at $r=0$, so we first establish a functional analytic setting for the equations. We exploit the Hilbert manifold structure of $\mathcal{E}_{g}$, and regard the first variation of energy as elements of the tangent Hilbert space

$$
\begin{gathered}
E=T \mathcal{E}_{g}=\left\{\left(u_{n}, v_{n}, \vec{a}\right): u_{n}, v_{n} \in H^{1}([-L, L]), \int_{-L}^{L} v_{0} \mathrm{~d} x=0,\right. \\
\left.\vec{a} \in H^{1}(D), \operatorname{div} \vec{a}=0 \text { in } D, \vec{a} \cdot \vec{n}=0 \text { on } \partial D\right\},
\end{gathered}
$$

and the second variation as a self-adjoint operator on $E$. We first introduce an equivalent inner product: for $\left(u_{n}, v_{n}, \vec{a}\right),\left(U_{n}, V_{n}, \vec{A}\right) \in E$, let

$$
\begin{aligned}
\left\langle\left(u_{n}, v_{n}, \vec{a}\right),\left(U_{n}, V_{n}, \vec{A}\right)\right\rangle:= & p \sum_{n=0}^{N} \int_{-L}^{L}\left\{2 u_{n} U_{n}+\frac{1}{\kappa^{2}}\left[u_{n}^{\prime} U_{n}^{\prime}+v_{n}^{\prime} V_{n}^{\prime}\right]+v_{n} V_{n}\right\} \mathrm{d} x \\
& +\frac{1}{\kappa^{2}} \iint_{D} \operatorname{curl} \vec{a} \cdot \operatorname{curl} \vec{A} \mathrm{~d} x \mathrm{~d} z .
\end{aligned}
$$

Then we define the gradient of $\Omega_{r}\left(f_{n}, \phi_{n}, \vec{A}\right)$ via

$$
\left\langle\nabla \Omega_{r}\left(f_{n}, \phi_{n}, \vec{A}\right),\left(u_{n}, v_{n}, \vec{a}\right)\right\rangle=D \Omega_{r}\left(f_{n}, \phi_{n}, \vec{A}\right)\left[u_{n}, v_{n}, \vec{a}\right] .
$$

PROPOSITION 2.1. - With $r=0$,

(a) $\inf \left\{\Omega_{0}\left(f_{n}, \phi_{n}, \vec{A}\right):\left(f_{n}, \phi_{n}, \vec{A}\right) \in \mathcal{E}_{g}\right\}=0$. The minimum value is attained, and the set of all minimizers coincides with the hyperplane $\mathcal{S}$, defined by the set of $\left(f_{n}, \phi_{n}, \vec{A}\right) \in \mathcal{E}_{g}$ such that: $f_{n} \equiv 1$;

$$
\begin{gathered}
\phi_{n}(x)=\alpha_{n}+\int_{0}^{x} A_{x}\left(s, z_{n}\right) \mathrm{d} s, \quad \alpha_{0}=0, \alpha_{n} \in \mathbf{R}, n=1, \ldots, N ; \\
\vec{A}=\left(\eta_{z},-\eta_{x}\right), \quad \text { where } \Delta \eta=H \text { in } D, \text { and }\left.\eta\right|_{\partial D}=0 .
\end{gathered}
$$

In particular, the gauge invariant phase difference is given by $\Phi_{n, n-1}^{0}=\delta_{n}+H p x$, where $\delta_{n}:=\alpha_{n}-\alpha_{n-1}, n=1, \ldots, N$. 
(b) For any element $s=\left(f_{n}^{0}, \phi_{n}^{0}, \vec{A}^{0}\right) \in \mathcal{S}$, the linearized operator $D^{2} \Omega_{0}(s): E \rightarrow E$ defines a Fredholm operator with index zero. Moreover,

$$
T_{S} \mathcal{S}=\operatorname{ker} D^{2} \Omega_{0}(s) \simeq \mathbf{R}^{N} .
$$

Note that $\mathcal{T}:=T_{S} \mathcal{S}$ is independent of $s \in \mathcal{S}$. Note that $\mathcal{S}$ may be parametrized by either $\left(\alpha_{1}, \ldots, \alpha_{N}\right)$ or $\left(\delta_{1}, \ldots, \delta_{N}\right) \in \mathbf{R}^{N} \simeq \mathcal{T}$. We abuse notation and write $s=$ $s\left(\alpha_{1}, \ldots, \alpha_{N}\right)$ or $s=s\left(\delta_{n}, \ldots, \delta_{N}\right)$ to emphasize the dependence.

Proof. - Being a sum of non-negative terms we clearly have $\inf _{\mathcal{E}_{g}} \Omega_{0}\left(f_{n}, \phi_{n}, \vec{A}\right) \geqslant 0$. The infimum of zero will be attained at $\left(f_{n}^{0}, \phi_{n}^{0}, \vec{A}^{0}\right)$ if and only if they solve the following first-order equations in our space $\mathcal{E}_{g}$ :

$$
f_{n}(x) \equiv 1, \quad \phi_{n}^{\prime}(x)-A_{x}\left(x, z_{n}\right) \equiv 0, \quad \operatorname{curl} \vec{A}=H .
$$

Note that by Lemma 1.4 the last equation is uniquely solved for $\vec{A}^{0} \in H^{1}(D)$, with solution as in (15). By the trace theorem $A_{x}\left(x, z_{n}\right) \in H^{1 / 2}([-L, L])$, and therefore, $\phi_{n}$ is uniquely determined by integration, except for the $(N+1)$ constants of integration $\alpha_{n}$. Note that $\eta$ is even in $x$, and hence $\int_{0}^{x} A_{x}^{0}\left(t, z_{n}\right) \mathrm{d} t$ is an odd function. In particular, $\alpha_{n}$ gives the average value of $\phi_{n}^{0}$ and thus $\alpha_{0}=0$ is fixed by the definition of the space $\mathcal{E}_{g}$, leaving $N$ free parameters $\left(\alpha_{1}, \ldots, \alpha_{N}\right)$ to parametrize the solution set. The explicit form for $\Phi_{n, n-1}^{0}$ is then obtained from (10). This completes the proof of (a).

Writing the linearized operator around a solution $\left(f_{n}^{0}, \phi_{n}^{0}, \vec{A}^{0}\right)$ as a quadratic form,

$$
\begin{aligned}
D^{2} & \Omega_{0}\left(f_{n}^{0}, \phi_{n}^{0}, \vec{A}^{0}\right)\left[u_{n}, v_{n}, \vec{a}\right] \\
& =p \sum_{n=0}^{N} \int_{-L}^{L}\left\{2 u_{n}^{2}+\frac{1}{\kappa^{2}}\left[u_{n}^{\prime}\right]^{2}+\frac{1}{\kappa^{2}}\left[v_{n}^{\prime}-a_{x}\left(x, z_{n}\right)\right]^{2}\right\} \mathrm{d} x+\frac{1}{\kappa^{2}} \iint_{D}|\operatorname{curl} \vec{a}|^{2} \mathrm{~d} x \mathrm{~d} z \\
& =\left\langle\left(u_{n}, v_{n}, \vec{a}\right),\left(u_{n}, v_{n}, \vec{a}\right)\right\rangle-p \sum_{n=0}^{N} \int_{-L}^{L}\left\{\frac{2}{\kappa^{2}} v_{n}^{\prime}(x) a_{x}\left(x, z_{n}\right)+v_{n}^{2}(x)\right\} \mathrm{d} x
\end{aligned}
$$

where $\left(u_{n}, v_{n}, \vec{a}\right) \in E$.

First note that by the second identity in (18), $D^{2} \Omega_{0}\left(f_{n}^{0}, \phi_{n}^{0}, \vec{A}^{0}\right)$ is of the form identity plus compact (since the trace embedding of $\vec{a} \rightarrow \vec{a}\left(\cdot, z_{n}\right)$ is compact from $H^{1}(D)$ to $\left.L^{2}([-L, L])\right)$. Next assume that $\left(u_{n}, v_{n}, \vec{a}\right) \in \operatorname{ker} D^{2} \Omega_{0}\left(f_{n}^{0}, \phi_{n}^{0}, \vec{A}^{0}\right)$. Clearly $u_{n} \equiv 0$ for all $n=0, \ldots, N$. By Lemma 1.5 we must have $\vec{a} \equiv 0$, and hence $v_{n}$ are constant. Since the definition of the space $E$ forces $v_{0}(x)$ with mean zero, we are left with $N$ free parameters, and

$$
\begin{aligned}
\operatorname{ker} D^{2} \Omega_{0}\left(f_{n}^{0}, \phi_{n}^{0}, \vec{A}^{0}\right)= & \left\{\left(u_{n}, v_{n}, \vec{a}\right) \in E:\left(u_{n}\right)_{n} \equiv 0, \vec{a} \equiv 0,\right. \\
& \left.\left(v_{0}, \ldots, v_{N}\right)=\left(0, c_{1}, \ldots, c_{N}\right), c_{1}, \ldots, c_{N} \in \mathbf{R}\right\} \\
= & T_{s} \mathcal{S} . \quad \square
\end{aligned}
$$




\section{Degenerate perturbation theory}

We now perturb away from the degenerate minima of $\Omega_{0}$, using a variational Lyapunov-Schmidt procedure. This method has been used by Ambrosetti, Coti-Zelati and Ekeland [3], Abrosetti and Badiale [2], Li and Nirenberg [19] (and many others) in a variety of situations involving heteroclinic solutions of Hamiltonian systems and in the semiclassical limit of the nonlinear Schrödinger equation.

We now proceed according to the usual Lyapunov-Schmidt reduction. Since $\mathcal{S}$ is a hyperplane, $\mathcal{T}=T_{s} \mathcal{S}$ is independent of $s \in \mathcal{S}$. Let $W=\mathcal{T}^{\perp}$, so any $\left(f_{n}, \phi_{n}, \vec{A}\right) \in \mathcal{E}_{g}$ admits the unique decomposition $\left(f_{n}, \phi_{n}, \vec{A}\right)=s+w$ with $s \in \mathcal{S}, w \in W$, and any $U:=\left(u_{n}, v_{n}, \vec{a}\right) \in E$ decomposes uniquely as $U=t+w$ with $t \in \mathcal{T}, w \in W$. We denote the orthogonal projection maps $P: E \rightarrow \mathcal{T}, P^{\perp}: E \rightarrow W$ so that $P U=t$, $P^{\perp} U=w$ whenever $U=t+w$. As in the previous section we interpret the first variation $\nabla \Omega_{r}\left(f_{n}, \phi_{n}, \vec{A}\right)$ as an element of $E$ itself, and project the equation $\nabla \Omega_{r}\left(f_{n}, \phi_{n}, \vec{A}\right)=0$ into the two linear subspaces $\mathcal{T}$ and $W$,

$$
\begin{aligned}
& F_{1}(r, s, w):=P\left[\nabla \Omega_{r}(s+w)\right]=0 ; \\
& F_{2}(r, s, w):=P^{\perp}\left[\nabla \Omega_{r}(s+w)\right]=0 .
\end{aligned}
$$

By Proposition 2.1(b), the second equation can be solved uniquely for $w=w(r, s)$ in a neighborhood of $\mathcal{S}$ for $r$ small, using the Implicit Function Theorem. Because our functional $\Omega_{r}$ is smooth we can expand $w(r, s)$ in powers of $r$. Note that $\Omega_{r}\left(s\left(\alpha_{1}, \ldots, \alpha_{N}\right)+w\right)$ is periodic in $\left(\alpha_{1}, \ldots, \alpha_{N}\right)$ so that we may think of $\mathcal{S}$ as a (compact) $N$-torus. Therefore the expansion will be uniform in $s$. We obtain the following variant of Lemma 2 of [2]:

LEMMA 3.1. - There exist constants $r_{0}>0$ and $\delta>0$, depending on $N, L, \kappa$, and $H$, and a smooth function

$$
w=w(r, s):\left(-r_{0}, r_{0}\right) \times \mathcal{S} \rightarrow W \subset E
$$

such that:

(i) There exists smooth functions $w_{1}, w_{2}$ such that

$$
w(r, s)=r w_{1}(s)+r^{2} w_{2}(r, s)
$$

for all $|r|<r_{0}$ and for all $s \in \mathcal{S}$;

(ii) $P^{\perp}\left[\nabla \Omega_{r}(s+w(r, s))\right]=0$.

(iii) Conversely, if $P^{\perp}\left[\nabla \Omega_{r}(s+w)\right]=0$ for some $r \in\left(-r_{0}, r_{0}\right)$ and $w \in W$ with $\|w\|_{E}<\delta$, then $w=w(r, s)$.

(iv) For any choice of $L_{0}, \kappa_{0}, H_{0}>0$ the constant $r_{0}$ may be chosen uniformly for all $N \geqslant 1,1 \leqslant L \leqslant L_{0}, 1 \leqslant \kappa \leqslant \kappa_{0}$, and $H \geqslant H_{0}$.

Parts (i)-(iii) follow easily from the Implicit Function Theorem. The dependences on the various parameters is more delicate: we provide the full proof in Section 2.5.

We define

$$
\mathcal{S}_{r}:=\{s+w(r, s): s \in \mathcal{S}\} \subset \mathcal{E}_{g} .
$$


$\mathcal{S}_{r}$ is a smooth manifold smoothly diffeomorphic to the hyperplane $\mathcal{S}$. The important role played by $\mathcal{S}_{r}$ is that it is a natural constraint for $\Omega_{r}$ (see Lemma 4 of [2]), and hence Eq. (19) may be solved variationally:

LEMMA 3.2.-

(a) If $\left(f_{n}, \phi_{n}, \vec{A}\right) \in \mathcal{S}_{r}$ satisfies $D\left(\Omega_{r \mid \mathcal{S}_{r}}\right)\left(f_{n}, \phi_{n}, \vec{A}\right)=0$, then $\nabla \Omega_{r}\left(f_{n}, \phi_{n}, \vec{A}\right)=0$ in $E$.

(b) There exists $\varepsilon_{0}=\varepsilon_{0}(N, L, \kappa, H)>0$ such that if $\left(f_{n}, \phi_{n}, \vec{A}\right) \in \mathcal{E}_{g}$ is a critical point of $\Omega_{r}$ with

$$
\Omega_{r}\left(f_{n}, \phi_{n}, \vec{A}\right) \leqslant \varepsilon_{0},
$$

then $\left(f_{n}, \phi_{n}, \vec{A}\right) \in \mathcal{S}_{r}$.

(c) There exists $\tilde{r}_{0}=\tilde{r}_{0}(N, L, \kappa, H)$ such that for all $0<r<\tilde{r}_{0}, \inf _{\mathcal{E}_{g}} \Omega_{r}=\inf _{\mathcal{S}_{r}} \Omega_{r}$.

Proof. - The assertion (a) is exactly Lemma 4 of [2]. To prove (b), assume that $\left(f_{n}, \phi_{n}, \vec{A}\right) \in \mathcal{E}_{g}$ is a critical point of $\Omega_{r}$ with energy bounded by (21). As remarked in the beginning of the section, $\left(f_{n}, \phi_{n}, \vec{A}\right)$ decomposes uniquely as $\left(f_{n}, \phi_{n}, \vec{A}\right)=s+w$, $s=\left(f_{n}^{0} \equiv 1, \phi_{n}^{0}, \vec{A}^{0}\right) \in \mathcal{S}, w \in W$. Now we use the energy bound to estimate the distance between $\left(f_{n}, \phi_{n}, \vec{A}\right)$ and $s$ : by Lemma 1.5 ,

$$
\begin{aligned}
& \left\|\vec{A}-\vec{A}_{0}\right\|_{H^{1}} \leqslant C_{0}\left\|\operatorname{curl} \vec{A}-\operatorname{curl} \vec{A}_{0}\right\|_{L^{2}}=C_{0}\|\operatorname{curl} \vec{A}-H\|_{L^{2}} \leqslant C_{0} \varepsilon_{0} \kappa^{2} ; \\
& p \sum_{n=0}^{N} \int_{-L}^{L}\left[\frac{1}{\kappa^{2}}\left(f_{n}^{\prime}\right)^{2}+\left(1-f_{n}\right)^{2}\right] \mathrm{d} x \leqslant p \sum_{n=0}^{N} \int_{-L}^{L}\left[\frac{1}{\kappa^{2}}\left(f_{n}^{\prime}\right)^{2}+\left(1-f_{n}^{2}\right)^{2}\right] \mathrm{d} x \leqslant \varepsilon_{0} ; \\
& p \sum_{n=0}^{N} \int_{-L}^{L} \frac{1}{\kappa^{2}}\left(\phi_{n}^{\prime}-\phi_{n 0}^{\prime}\right)^{2} \mathrm{~d} x \\
& \quad=p \sum_{n=0}^{N} \int_{-L}^{L} \frac{1}{\kappa^{2}}\left(\phi_{n}^{\prime}-A_{x 0}\left(x, z_{n}\right)\right)^{2} \mathrm{~d} x \\
& \leqslant 2 p \sum_{n=0}^{N} \int_{-L}^{L}\left(\frac{1}{\kappa^{2}}\left(\phi_{n}^{\prime}-A_{x}\left(x, z_{n}\right)\right)^{2}+\frac{1}{\kappa^{2}}\left(A_{x}\left(x, z_{n}\right)-A_{x 0}\left(x, z_{n}\right)\right)^{2}\right) \mathrm{d} x \\
& \leqslant 2 \varepsilon_{0}+C_{1}\left\|\vec{A}-\vec{A}_{0}\right\|_{H^{1}} \leqslant C_{2} \varepsilon_{0},
\end{aligned}
$$

where $C_{1}$ comes from applying the Trace theorem (see Lemma 5.2 in Section 5) in the last line. Finally, since $\left[\left(f_{n}, \phi_{n}, \vec{A}\right)-s\right] \in W$, each $\phi_{n}-\phi_{n}^{0}$ has mean value zero. Therefore, the $H^{1}([-L, L])$-norm of the difference is controlled by the difference of the derivatives, as estimated above, and we may choose $\varepsilon_{0}$ small enough such that

$$
\operatorname{dist}\left(\left(f_{n}, \phi_{n}, \vec{A}\right),\left(f_{n 0}, \phi_{n 0}, \vec{A}_{0}\right)\right)<c \varepsilon_{0}<\delta,
$$

where $\delta=\delta(N, L, \kappa, H)$ is given by Lemma 3.1. It then follows by assertion (iii) of Lemma 3.1 that if $\left(f_{n}, \phi_{n}, \vec{A}\right)$ is a critical point of the Lawrence-Doniach system with the given energy bound it must lie on $\mathcal{S}_{r}$. This completes the proof of (b). 
To prove (c) we note that Proposition 1.1 implies that $\inf _{\mathcal{E}_{g}} \Omega_{r} \leqslant 2 N p\left(L+\frac{1}{H p}\right) r$, and hence we can choose $\tilde{r}_{0}<r_{0}$ such that $2 N p\left(L+\frac{1}{H p}\right) \tilde{r}_{0} \leqslant \varepsilon_{0}$.

Remark 3.3. - We note that we cannot make the same statement about the $N, L, \kappa, H$ dependences of $\tilde{r}_{0}$ in (c) of Proposition 3.2 as we make for $r_{0}$ in Lemma 3.1. The uniform bounds on $r_{0}$ are possible because of local estimates on the solution set of (20) and a continuity argument from $r=0$. (See Section 5.) We have no such control on the distance $\delta$ from $\mathcal{S}$ to solutions which do not lie on on the manifold $\mathcal{S}_{r}$. This would entail uniform (in the parameters) global (i.e., non-perturbative) information about the solution set, which energy bounds do not provide. This leaves open the possibility of an interval $r \in\left(\tilde{r}_{0}, r_{0}\right)$ for which the solutions on $\mathcal{S}_{r}$ continue to exist and are represented by a perturbation expansion in $r$, but the absolute minimizer might not be an element of this family.

In conclusion, we have achieved a complete finite-dimensional reduction of our problem, for small $r$. That is to say, when $0<r<\tilde{r}_{0}$ all low energy solutions of the Lawrence-Doniach system can be found on the $N$-dimensional surface $\mathcal{S}_{r}$. Moreover, an explicit form for these solutions may be determined by a simple procedure of Taylor expansion of the equations and energy in powers of $r$, as is legitimized by Lemma 3.1.

\section{Vortex planes}

We now apply the theory of the previous section to determine the minimizer (and other stationary states) of the Lawrence-Doniach energy, for $r \ll 1$.

We summarize our results in the following:

THEOREM 4.1. - Assume that $\sin (H p L) \neq 0$. There exists $r_{1}=r_{1}(N, L, \kappa, H)$ such that for every $r$ with $0<r<r_{1}$, the global minimum of free energy is attained by the vortex plane solutions, given asymptotically by (28)-(34) below. Moreover, when $0<r<r_{1} \Omega_{r}$ admits exactly $2^{N}$ physically distinct critical points with energy bounded as in (21).

\subsection{Minimizing $\left.\Omega_{r}\right|_{\mathcal{S}_{r}}$}

By Proposition 3.2 we seek critical points of the finite dimensional variational problem $\left.\Omega_{r}\right|_{\mathcal{S}_{r}}$. Using Lemma 3.1(i) we expand a point $\left(f_{n}, \phi_{n}, \vec{A}\right) \in \mathcal{S}_{r}$ as

$$
\left(f_{n}, \phi_{n}, \vec{A}\right)=s+w(r, s)=\left(f_{n}^{0}, \phi_{n}^{0}, \vec{A}^{0}\right)+r\left(u_{n}, v_{n}, \vec{a}\right)+\mathrm{O}\left(r^{2}\right),
$$

where $\left(f_{n}^{0}, \phi_{n}^{0}, \vec{A}^{0}\right) \in \mathcal{S}$ solve the first-order system (17), and the error term is uniform over $s \in \mathcal{S}$. We observe that $\Omega_{r}$ has the form,

$$
\begin{aligned}
\Omega_{r}\left(f_{n}, \phi_{n}, \vec{A}\right) & =\Omega_{0}\left(f_{n}, \phi_{n}, \vec{A}\right)+r \Gamma\left(f_{n}, \phi_{n}, \vec{A}\right), \quad \text { with } \\
\Gamma\left(f_{n}, \phi_{n}, \vec{A}\right) & =\frac{p}{2} \sum_{n=1}^{N} \int_{-L}^{L}\left(f_{n}^{2}+f_{n-1}^{2}-2 f_{n} f_{n-1} \cos \left(\Phi_{n, n-1}\right)\right) \mathrm{d} x .
\end{aligned}
$$


Since $\Omega_{r}(s+w(r, s))$ is a smooth function of $s=s\left(\delta_{1}, \ldots, \delta_{N}\right)$ and $r$, it admits a Taylor expansion of the form,

$$
\begin{aligned}
\Omega_{r}(s+w(r, s)) & =\Omega_{0}(s)+\left.r \frac{\partial}{\partial r} \Omega_{r}(s+w(r, s))\right|_{r=0}+\mathrm{O}\left(r^{2}\right) \\
& =r\left(\Gamma(s)+\nabla \Omega_{0}(s)\left[\frac{\partial w}{\partial r}(0, s)\right]\right)+\mathrm{O}\left(r^{2}\right)=r \Gamma(s)+\mathrm{O}\left(r^{2}\right) \\
& =r p \sum_{n=1}^{N} \int_{-L}^{L}\left[1-\cos \left(\delta_{n}+H p x\right)\right] \mathrm{d} x+\mathrm{O}\left(r^{2}\right) \\
& =2 p\left(N L-\frac{\sin (H p L)}{H p} \sum_{n=1}^{N} \cos \delta_{n}\right) r+\mathrm{O}\left(r^{2}\right),
\end{aligned}
$$

with remainder term uniform for $s \in \mathcal{S}$.

Define $G: \mathbf{R} \times \mathbf{R}^{N} \rightarrow \mathbf{R}$ by:

$$
G\left(r, \delta_{1}, \ldots, \delta_{N}\right):=\Omega_{r}(s+w(r, s)) / r, \quad s=s\left(\delta_{1}, \ldots, \delta_{N}\right) .
$$

Then $G$ is a smooth function which is periodic in each coordinate $\delta_{n}$, and

$$
G\left(0, \delta_{1}, \ldots, \delta_{N}\right)=2 N p L-\frac{2 \sin (H p L)}{H} \sum_{n=1}^{N} \cos \delta_{n} .
$$

Note that when

$$
\sin (H p L) \neq 0
$$

$G(0, s)$ possesses exactly $2^{N}$ critical points for $\left(\delta_{1}, \ldots, \delta_{n}\right) \in \mathcal{K}:=\mathbf{R}^{N} /(2 \pi \mathbf{Z})^{N}$ corresponding to any permutation of

$$
\delta_{n} \in\{0, \pi\} \bmod 2 \pi
$$

It is easy to see that each is a non-degenerate critical point of $G(0, s)$. By the Implicit Function Theorem, there exists $\tilde{r}_{1}>0$ such that for all $r \in\left(0, \tilde{r}_{1}\right)$, and for any critical point $\left(\delta_{1}^{*}, \ldots, \delta_{N}^{*}\right)$ of $G\left(0, \delta_{1}, \ldots, \delta_{N}\right)$ there exists a unique critical point $\left(\delta_{1}(r), \ldots, \delta_{N}(r)\right)$ of $G\left(r, \delta_{1}, \ldots, \delta_{N}\right)$, with

$$
\left(\delta_{1}(r), \ldots, \delta_{N}(r)\right)=\left(\delta_{1}^{*}, \ldots, \delta_{N}^{*}\right)+\mathrm{O}(r) .
$$

Since $G$ is periodic in each $\delta_{n}$ we may also conclude (via a compactness argument) that these are the only critical points of $G\left(r, \delta_{1}, \ldots, \delta_{N}\right)$ for small $r$. Since $\left.\Omega_{r}\right|_{\mathcal{S}_{r}}=$ $r G\left(r, \delta_{1}, \ldots, \delta_{N}\right)$, by Lemma 3.2, therefore, for all $r$ with $0<r<\min \left\{\tilde{r}_{0}, \tilde{r}_{1}\right\}:=r_{1}$ and whenever $\sin (H p L) \neq 0, \Omega_{r}$ admits exactly $2^{N}$ critical points $\left(\bmod 2 \pi\right.$ in each $\left.\phi_{n}(x)\right)$ with energy bound (21). 
The absolute minimizer of $G(0, s)$ is obtained for

$$
\delta_{n}^{*}= \begin{cases}0, & \text { when } \frac{\sin (H p L)}{H p}>0, \text { for every } n=1, \ldots, N ; \\ \pi, & \text { when } \frac{\sin (H p L)}{H p}<0, \text { for every } n=1, \ldots, N .\end{cases}
$$

By the Implicit Function Theorem argument in the previous paragraph, we conclude that for all $r \in\left(0, r_{1}\right),\left.\Omega_{r}\right|_{\mathcal{S}_{r}}$ is minimized by a unique $s_{r}=s\left(\delta_{1}(r), \ldots, \delta_{N}(r)\right)$ with

$$
\delta_{n}(r)=\delta^{*}+\mathrm{O}(r), \quad n=1, \ldots, N,
$$

where $\delta^{*} \in\{0, \pi\}$ is chosen as in (24). Finally, by Lemma 3.2 we conclude that for all $r \in\left(0, r_{1}\right)$ and $\sin (H p L) \neq 0$ the absolute minimizer of $\Omega_{r}$ in $\mathcal{E}_{g}$ is given by $\left(f_{n}, \phi_{n}, \vec{A}\right)=s_{r}+r w_{1}\left(s_{r}\right)+\mathrm{O}\left(r^{2}\right)$, with minimum energy

$$
\inf _{\mathcal{E}_{g}} \Omega_{r}=2 N p\left(L-\frac{\sin (H p L}{H p}\right) r+\mathrm{O}\left(r^{2}\right) .
$$

When $\sin (H p L)=0$ then $\Omega_{r \mid \mathcal{S}_{r}}$ is degenerate at order $r$. Normally we should go to a higher order in the expansion to determine the stationary configurations at these exceptional values of $H=m \pi / L p, m=1,2,3, \ldots$, but (as we will see later in the section) the existence of these degenerate points is explained by the exchange of stability of two families of solutions when vortices are nucleated into the sample from the boundary. We note that the treatment of the periodic problem in the subsequent paper [1] will require an expansion of the energy to order $r^{2}$ to resolve the degeneracy at any applied field $H$.

\subsection{Expanding the solutions to order $r$}

Because $\Omega_{r}$ is a smooth functional we may use the Implicit Function Theorem and the decomposition of Lemma 3.1 to obtain an expansion to arbitrary order in $r$ of any critical point satisfying the energy estimate (21). Here we generate the expansion to order $r$, to get a better idea of the nature of the global minimizers. Take any such critical point of $\left.\Omega_{r}\right|_{\mathcal{S}_{r}}$, with expansion as in (i) of Lemma 3.1, $\left(f_{n}, \phi_{n}, \vec{A}\right)=s+r w_{1}(s)+\mathrm{O}\left(r^{2}\right)$, $s=s\left(\delta_{1}, \ldots, \delta_{N}\right)$. We deduce the equation satisfied by $w_{1}(s)$ by implicit differentiation of Eq. (20):

$$
\begin{aligned}
0 & =\left.\frac{\mathrm{d}}{\mathrm{d} r} P^{\perp}\left(\nabla \Omega_{r}(s+w(r, s))\right)\right|_{r=0} \\
& =P^{\perp}\left(\nabla \Gamma(s)+D^{2} \Omega_{0}(s)\left[\frac{\partial w}{\partial r}(0, s)\right]\right) \\
& =P^{\perp}\left(\nabla \Gamma(s)+D^{2} \Omega_{0}(s)\left[w_{1}(s)\right]\right) .
\end{aligned}
$$

Since $D^{2} \Omega_{0}(s)$ is an invertible map from $W \rightarrow W$ this formula uniquely determines $w_{1}(s)$. Now we represent $w_{1}(s)=\left(u_{n, 1}, v_{n, 1}, a_{x, 1}, a_{z, 1}\right)$, in other words

$$
\begin{aligned}
f_{n} & =1+r u_{n, 1}+\mathrm{O}\left(r^{2}\right), \\
A_{x} & =H z+r a_{x, 1}+\mathrm{O}\left(r^{2}\right), \quad \phi_{n}=\alpha_{n}+n H p x+v_{n, 1}+\mathrm{O}\left(r^{2}\right), \\
A_{z} & =r a_{z, 1}+\mathrm{O}\left(r^{2}\right),
\end{aligned}
$$


and denote $b(x, z)=\operatorname{curl} \vec{a}_{1}=\partial_{z} a_{x, 1}-\partial_{x} a_{z, 1}$. In terms of these coordinates (25) takes the following simple form:

$$
-\frac{1}{\kappa^{2}} u_{n, 1}^{\prime \prime}+2 u_{n, 1}=\frac{1}{2} \begin{cases}\cos \left(\delta_{n}+H p x\right)+\cos \left(\delta_{n+1}+H p x\right)-2, & 1 \leqslant n \leqslant N-1, \\ \cos \left(\delta_{N}+H p x\right)-1, & n=N, \\ \cos \left(\delta_{1}+H p x\right)-1, & n=0,\end{cases}
$$

with $u_{n, 1}^{\prime}( \pm L)=0$;

$$
\begin{aligned}
& \frac{1}{\kappa^{2}} \frac{\mathrm{d}}{\mathrm{d} x}\left(v_{n, 1}^{\prime}-a_{x, 1}\left(x, z_{n}\right)\right) \\
& \quad=\frac{1}{2} \begin{cases}\sin \left(\delta_{n}+H p x\right)-\sin \left(\delta_{n+1}+H p x\right)-I_{n}, & 1 \leqslant n \leqslant N-1, \\
\sin \left(\delta_{N}+H p x\right)-I_{N}, & n=N, \\
-\sin \left(\delta_{1}+H p x\right)-I_{0}, & n=0,\end{cases}
\end{aligned}
$$

with boundary condition $v_{n, 1}^{\prime}( \pm L)-a_{x, 1}\left( \pm L, z_{n}\right)=0$ and

$$
I_{n}=\frac{1}{2 L} \begin{cases}\int_{-L}^{L}\left[\sin \left(\delta_{n}+H p x\right)-\sin \left(\delta_{n+1}+H p x\right)\right] \mathrm{d} x, & 1 \leqslant n \leqslant N-1, \\ \int_{-L}^{L} \sin \left(\delta_{N}+H p x\right) \mathrm{d} x, & n=N, \\ -\int_{-L}^{L} \sin \left(\delta_{1}+H p x\right) \mathrm{d} x, & n=0\end{cases}
$$

and

$$
\vec{a}_{1}(x, z)=\left(\frac{\partial \xi}{\partial z},-\frac{\partial \xi}{\partial x}\right), \quad \Delta \xi=b(x, z),\left.\quad \xi\right|_{\partial B}=0
$$

where $b(x, z)=b^{(n)}(x)$ for $z_{n-1}<z<z_{n}$, with

$$
\frac{\partial b^{(n)}}{\partial x}=\frac{p \kappa^{2}}{2}\left[\sin \left(\delta_{n}+H p x\right)-\frac{1}{2 L} \int_{-L}^{L} \sin \left(\delta_{n}+H p x\right) \mathrm{d} x\right], \quad b^{(n)}( \pm L)=0,
$$

for $n=1, \ldots, N$.

Now assume that $\sin (H p L) \neq 0$ and consider the absolute minimizers, $\delta_{n} \equiv \delta_{*}$ with $\delta_{*} \in\{0, \pi\}$ chosen according to (24). Except for an edge effect at the top and bottom layers $(n=0, N)$ the gauge-invariant quantities are independent of $n$ at order $r$. In particular, the magnetic field $h$, and Josephson current density $j_{z}^{(n)}$ are (at order $r$ ) completely independent of $z, n$ :

$$
\begin{aligned}
& j_{z}^{(n)}:=j_{c} f_{n} f_{n-1} \sin \Phi_{n, n-1}=r \frac{\kappa^{2} p}{2} \sin (\delta+H p x)+\mathrm{O}\left(r^{2}\right), \\
& h(x, z)=H+r \frac{\kappa^{2}}{2 H}(\cos (\delta+H p L)-\cos (\delta+H p x))+\mathrm{O}\left(r^{2}\right)
\end{aligned}
$$

where $j_{c}=r \frac{\kappa^{2} p}{2}$ is the critical Josephson current. The in-plane supercurrent vanishes at order $r$ for all interior planes,

$$
j_{x}^{(n)}=\mathrm{O}\left(r^{2}\right), \quad n=1, \ldots, N-1,
$$


but the top and bottom of the sample carry current at order $r$ :

$$
\begin{aligned}
& j_{x}^{(N)}=0+r \frac{\kappa^{2}}{2 H p}(\cos (\delta+H p L)-\cos (\delta+H p x))+\mathrm{O}\left(r^{2}\right), \\
& j_{x}^{(0)}=0+r \frac{\kappa^{2}}{2 H p}(\cos (\delta+H p x)-\cos (\delta+H p L))+\mathrm{O}\left(r^{2}\right) .
\end{aligned}
$$

Similarly, the order $r$ expansion of modulus of the order parameter is $n$-independent on the interior planes, but is modified at the top and bottom at order $r$ :

$$
\begin{gathered}
f_{n}(x)=1+r\left(-\frac{1}{2}+A \cosh \left(\frac{\sqrt{2}}{\kappa} x\right)+B \cos (\delta+H p x)\right)+\mathrm{O}\left(r^{2}\right), \\
n=1, \ldots, N-1 \\
f_{0}=f_{N}=1+\frac{r}{2}\left(-\frac{1}{2}+A \cosh \left(\frac{\sqrt{2}}{\kappa} x\right)+B \cos (\delta+H p x)\right)+\mathrm{O}\left(r^{2}\right),
\end{gathered}
$$

where

$$
A=\frac{\kappa^{3} H p}{\sqrt{2}\left(H^{2} p^{2}+2 \kappa^{2}\right)} \frac{\sin (\delta+H p L)}{\sinh \left(\frac{\sqrt{2} L}{\kappa}\right)}, \quad B=\frac{\kappa^{2}}{H^{2} p^{2}+2 \kappa^{2}} .
$$

Expansion to order $r^{2}$ in the solutions will show these quantities to be independent of $z$ (or $n$ ) except for the top and bottom two planes and the top and bottom gaps.

We note that the gauge-invariant phase difference,

$$
\Phi_{n, n-1}(x)=\delta+H p x+r \frac{\kappa^{2}}{2 H^{2}}\left(x H p \cos (\delta+H p L)-\sin (\delta+H p x)+c_{n}\right)+\mathrm{O}\left(r^{2}\right),
$$

contains constants $c_{n}$ which can only be determined by higher order expansion in $r$. Indeed, they represent the order $r$ correction to the choice of $\delta_{n}$ when minimizing the finite dimensional problem $\left.\Omega_{r}\right|_{\mathcal{S}_{r}}$.

We call this configuration vortex planes - see Fig. 1. Unlike the Ginzburg-Landau case, the order parameter does not vanish at the "core" where the local field attains its maximal value and around which supercurrents circulate. The "vortices" are then the planes $\{x=$ const. $\}$ over which $h(x)$ attains its relative maxima. These planes are nodes for the current, and flux per plane per cycle of the Josephson current is, to order $r$, the usual flux quantum $(2 \pi$ in our units).

Remark 4.2.- (a) The distinction between the energies of the various lattice geometries at the lowest order term $\mathrm{O}(r)$ in expansion (23) is a surface term, in the sense that it scales like the length of the lateral edges of the sample $2 \mathrm{~Np}$, as opposed to the free energy itself which scales like the cross-sectional area of the bulk $2 L N p$. In other words, the vortex plane configuration is preferred for the effect it has on the surface currents on the left and right edges of the sample, with no regard to energy minimization in the interior. As we will see in the periodic case in [1] the effect on the bulk will be observed in an order $r^{2}$ term. For all other parameters fixed, eventually we can take $r>0$ small enough so that the $\mathrm{O}(r)$ surface term dominates. But in a real superconductor the 
value of $r$ is given, and hence when the width $L$ is increased eventually the bulk $\mathrm{O}\left(r^{2}\right)$ term will compete with the surface $\mathrm{O}(r)$ term. This suggests that the interval of validity of the $r$ expansion may not be uniform, but rather deteriorate with increasing sample width $L$.

(b) Another indication that the radius of convergence of the expansion depends inversely on the width $L$ is the presence of a linearly growing factor (secular term) in the order $r$ term of expansion (34) of $\Phi_{n, n-1}(x)$.

\subsection{Vortex plane nucleation}

The very precise desciption of minimizers for $r \simeq 0$ allows us to identify the transitions which the sample undergoes as new vortices are nucleated in an increasing applied field $H$. When $0 \leqslant H<\pi / p L$ we observe the Meissner state for this problem: $h$ attains its maximum at the boundary $x= \pm L$, and decreases to a line of minima at $x=0$. For our solutions the lower critical field $H_{C 1}=\pi / p L$ : at this point the finite dimensional minimization problem $G(0, s)=0$ degenerates, and when $H$ is increased slightly the minimizing configuration has phases $\delta_{n}=\pi$ for all $n=1, \ldots, N$. Note that at this critical value of $H$ a new node appears in the Josephson current (28) at each endpoint. The switch in $\delta_{n}$ amounts to a flip in sign for the Josephson currents and for the variation of $h$ from $H$, so the newly nucleated nodes correspond to minima of $h$, with the former minimum at $x=0$ becoming a local maximum. The same phenomenon will occur every time $H$ crosses a value $\frac{k \pi}{p L}, k=2,3, \ldots$ Each time, two new nodes for the Josephson current will be nucleated, and the change of $\delta_{n}$ from zero to $\pi$ (or viceversa) will exchange minima and maxima of $h$ in the interior, resulting in the creation of exactly one new vortex plane. Since the minimum energy is given asymptotically by

$$
\varepsilon(H):=\min \Omega_{r}=\operatorname{Npr}\left(2 L-\left|\frac{2 \sin (H p L)}{H p}\right|\right)+\mathrm{O}\left(r^{2}\right),
$$

the magnetization $M(H):=\partial \varepsilon / \partial H$ is discontinuous at each nucleation, indicating a first-order phase transition.

\section{The validity of the expansion}

In this section we prove Lemma 3.1 which justifies the finite dimensional reduction in a neighborhood of $\mathcal{S}$. While parts (i)-(iii) follow easily from the Implicit Function Theorem, the real interest is in the dependence of the interval of validity $|r|<r_{0}$ on the many parameters in the model, especially the sample dimensions $L$ and $M=N p, \kappa$, and the applied field $H$. Here we give a lower bound for the "radius of convergence" in $r$ as a function of these parameters. We show that the small $r$ approximation is essentially independent of the number of planes $N$, but that its validity can be expected to decrease with increasing width $L$ or increasing $\kappa$. On the other hand, increasing the external field $H$ enhances the approximations somewhat. This lower bound is consistent with experiments on the high- $T_{c}$ materials, where the vortex planes have not been observed, perhaps because the large values of $\kappa$ and the large size of typical samples (measured in terms of $\lambda_{a b}$ ) reduce the radius of validity of the expansion below the appropriate value 
of $r$ for such materials. For other types of layered superconductors with smaller values of $\kappa$ we are more likely to see configurations similar to the solutions produced in our $r \rightarrow 0$ limit.

Throughout this section, we concentrate on the parameters $N, L, \kappa, H$, and assume $0<p<1, \kappa \geqslant 1$, and $L \geqslant 1$. We also define a norm on the space $W=[T \mathcal{S}]^{\perp}$ : let $w=\left(u_{n}, v_{n}, \vec{a}\right) \in W$. Then we denote

$$
\begin{aligned}
\|w\|^{2}=\|\left[\left(u_{n}, v_{n}, \vec{a}\right) \|^{2}=\right. & p \sum_{n=0}^{N} \int_{-L}^{L}\left(\left(u_{n}^{\prime}\right)^{2}+u_{n}^{2}+\left(v_{n}^{\prime}\right)^{2}+v_{n}^{2}\right) \mathrm{d} x \\
& +\iint_{D}\left[|\nabla \vec{a}|^{2}+|\vec{a}|^{2}\right] \mathrm{d} x \mathrm{~d} z .
\end{aligned}
$$

Our approach is to calculate a priori estimates on the solutions to Eq. (20) to determine when that equation can degenerate. As a first step, we must recognize the system of differential equations satisfied by the solutions to (20). For any $U=\left(f_{n}, \phi_{n}, \vec{A}\right)$ we write $U=s+w(r, s)$, where $s=s\left(\delta_{1}, \ldots, \delta_{N}\right) \in \mathcal{S}$, and $w(r, s)=\left(u_{n}, v_{n}, \vec{a}\right) \in W$. We recall the effect that this decomposition has on some familiar quantities:

$$
\begin{gathered}
f_{n}=1+u_{n}, \quad V_{n}(x)=\phi_{n}^{\prime}-A_{x}\left(x, z_{n}\right)=v_{n}^{\prime}-a_{x}\left(x, z_{n}\right), \\
\vec{a}(x, z)=\left(\partial_{z} \xi,-\partial_{x} \xi\right), \quad \Delta \xi=b(x, z),\left.\quad \xi\right|_{\partial D}=0, \quad h(x, z)=H+b(x, z), \\
\Phi_{n, n-1}=\phi_{n}-\phi_{n-1}-\int_{z_{n-1}}^{z_{n}} A_{z}(x, t) \mathrm{d} t=H p x+\delta_{n}+\varphi_{n, n-1}, \\
\text { where } \varphi_{n, n-1}(x)=v_{n}(x)-v_{n-1}(x)-\int_{z_{n-1}}^{z_{n}} a_{z}(x, t) \mathrm{d} t .
\end{gathered}
$$

The equations for $\left(u_{n}, v_{n}, \vec{a}\right)$ will differ from the unconstrained Euler-Lagrange equations derived in Section 2 because of Lagrange multipliers created by projection into the subspace $W$.

To say $w(r, s)$ solves $P^{\perp} D \Omega_{r}(s+w(r, s))=0$ is equivalent to saying that $\Omega_{r}(s+$ $w(r, s))$ is stationary with respect to variations in $W$. Taking the variation with respect to just one of the $u_{n}$ yields the exact same Eqs. (2) as the unconstrained case, which we rewrite in terms of $u_{n}$ :

$$
\begin{aligned}
& -\frac{1}{\kappa^{2}} u_{n}^{\prime \prime}+\left(u_{n}+1\right)\left(u_{n}+2\right) u_{n}+\frac{1}{\kappa^{2}}\left(v_{n}^{\prime}-a_{x}\left(x, z_{n}\right)\right)^{2} u_{n}=-\frac{1}{\kappa^{2}}\left(v_{n}^{\prime}-a_{x}\left(x, z_{n}\right)\right)^{2} \\
& \quad+ \begin{cases}\frac{r}{2}\left(\left(1+u_{n-1}\right) \cos \Phi_{n, n-1}+\left(1+u_{n+1}\right) \cos \Phi_{n+1, n}-2\left(1+u_{n}\right)\right), & n \neq 0, N ; \\
\frac{r}{2}\left(\left(1+u_{1}\right) \cos \Phi_{1,0}-\left(1+u_{0}\right)\right), & n=0 ; \\
\frac{r}{2}\left(\left(1+u_{N-1}\right) \cos \Phi_{N, N-1}-\left(1+u_{N}\right)\right), & n=N,\end{cases}
\end{aligned}
$$

with boundary conditions $u_{n}^{\prime}( \pm L)=0$.

For $v_{n}, n=0, \ldots, N$, the integral constraint gives rise to the usual Lagrange multiplier, without which the equations generally could not be integrated: 


$$
\begin{aligned}
& \frac{1}{\kappa^{2}} \frac{\mathrm{d}}{\mathrm{d} x}\left(f_{n}^{2}\left(v_{n}^{\prime}-a_{x}\left(x, z_{n}\right)\right)\right) \\
& \quad= \begin{cases}\frac{r}{2}\left[f_{n} f_{n-1} \sin \Phi_{n, n-1}-f_{n+1} f_{n} \sin \Phi_{n+1, n}\right]-C_{n}, & n=1, \ldots, N-1 ; \\
\frac{r}{2} f_{N} f_{N-1} \sin \Phi_{N, N-1}-C_{N}, & n=N, \\
-\frac{r}{2} f_{0} f_{1} \sin \Phi_{1,0}+C_{0}, & n=0,\end{cases}
\end{aligned}
$$

where

$$
C_{n}= \begin{cases}\frac{1}{2 L} \int_{-L}^{L} \frac{r}{2}\left[f_{n} f_{n-1} \sin \Phi_{n, n-1}-f_{n+1} f_{n} \sin \Phi_{n+1, n}\right] \mathrm{d} x, & n=1, \ldots, N-1 ; \\ \frac{1}{2 L} \int_{-L}^{L} \frac{r}{2} f_{N} f_{N-1} \sin \Phi_{N, N-1} \mathrm{~d} x, & n=N, \\ \frac{1}{2 L} \int_{-L}^{L} \frac{r}{2} f_{0} f_{1} \sin \Phi_{1,0} \mathrm{~d} x, & n=0 .\end{cases}
$$

The equations for $v_{n}$ also include a no-flux boundary condition,

$$
\left.f_{n}^{2}\left(v_{n}^{\prime}-a_{x}\left(\cdot, z_{n}\right)\right)\right|_{x= \pm L}=0, \quad n=0, \ldots, N .
$$

Finally, we derive the Euler-Lagrange equations for $\vec{a}$. As in our previous calculations it will be easier to deal with the associated magnetic field $b(x, z)=\operatorname{curl} \vec{a}(x, z)$.

LEMMA 5.1. - If $\left(f_{n}, \phi_{n}, \vec{A}\right)=s+w(r, s)$ satisfies (20), then $b(x, z)=h(x, z)-H$ satisfies:

$$
\begin{gathered}
b(x, z)=b^{(n)}(x), \quad z_{n-1}<z<z_{n}, n=1, \ldots, N, \\
b^{(n)}(x)-b^{(n+1)}(x)=p\left(v_{n}^{\prime}-a_{x}\left(x, z_{n}\right)\right), \quad n=1, \ldots, N-1, \\
b^{(N)}(x)=p\left(\left(v_{N}^{\prime}-a_{x}\left(x, z_{N}\right)\right) f_{N}^{2}\right), \quad b^{(1)}(x)=-p\left(\left(v_{0}^{\prime}-a_{x}\left(x, z_{0}\right)\right) f_{0}^{2}\right), \\
\frac{\mathrm{d} b^{(n)}}{\mathrm{d} x}=\frac{r p \kappa^{2}}{2} f_{n} f_{n-1} \sin \Phi_{n, n-1}(x)-D_{n}, \quad b^{(n)}( \pm L)=0, \\
\text { where } D_{n}=\frac{1}{2 L} \int_{-L}^{L} \frac{r p \kappa^{2}}{2} f_{n} f_{n-1} \sin \Phi_{n, n-1}(x) \mathrm{d} x .
\end{gathered}
$$

Proof. - First we derive the equation for $\vec{a}$ in the weak form. Any admissible variation for $\vec{a}$ in $E$ can be represented in the form $\tilde{a}=\left(\partial_{z} \eta,-\partial_{x} \eta\right)$ with $\eta \in H^{2} \cap H_{0}^{1}(D)$. (Indeed, $\eta$ solves $\Delta \eta=\operatorname{curl} \tilde{a}$ in with $\left.\eta\right|_{\partial D}=0$ : see Lemma 1.5.) Taking the first variation of energy in the direction of $\tilde{a}$ we obtain:

$$
\begin{aligned}
0=- & p \sum_{n=0}^{N} \int_{-L}^{L} \frac{1}{\kappa^{2}}\left(v_{n}^{\prime}-a_{x}\left(x, z_{n}\right)\right) f_{n}^{2} \partial_{z} \eta\left(x, z_{n}\right) \mathrm{d} x \\
& +\sum_{n=1}^{N} \int_{-L z_{n-1}}^{L} \int_{z_{n}}^{z_{n}}\left\{\frac{1}{\kappa^{2}} b(x, z) \Delta \eta+\frac{r p}{2} f_{n} f_{n-1} \sin \Phi_{n, n-1} \partial_{x} \eta\right\} \mathrm{d} z \mathrm{~d} x,
\end{aligned}
$$

for all $\eta \in H^{2} \cap H_{0}^{1}(D)$. 
Next, we observe that there exist functions $\hat{b}^{n}(x) \in H^{1}(-L, L)$ which solve the system (38)-(40). Indeed, we define $\hat{b}^{n}$ by integration,

$$
\hat{b}^{n}(x)=\int_{-L}^{x}\left(\frac{r p \kappa^{2}}{2} f_{n}(x) f_{n-1}(x) \sin \Phi_{n, n-1}(x)-D_{n}\right) \mathrm{d} x, \quad n=1, \ldots, N .
$$

Then $\hat{b}^{n}(x) \in H^{1}([-L, L])$ and satisfies (40). For $n=1, \ldots, N-1$ we have:

$$
\begin{aligned}
\frac{\mathrm{d}}{\mathrm{d} x}\left(\hat{b}^{n+1}(x)-\hat{b}^{n}(x)\right) & =\frac{r p \kappa^{2}}{2}\left(f_{n+1} f_{n} \sin \Phi_{n+1, n}-f_{n} f_{n-1} \sin \Phi_{n, n-1}\right)-D_{n+1}+D_{n} \\
& =-p \frac{\mathrm{d}}{\mathrm{d} x}\left(\left(v_{n}^{\prime}-a_{x}\left(x, z_{n}\right)\right) f_{n}^{2}\right),
\end{aligned}
$$

by (36). For the top and bottom gaps $(n=1, N)$ we obtain:

$$
\begin{aligned}
\frac{\mathrm{d}}{\mathrm{d} x} \hat{b}^{N}(x) & =\frac{r p \kappa^{2}}{2} f_{N} f_{N-1} \sin \Phi_{N, N-1}-D_{N}=p \frac{\mathrm{d}}{\mathrm{d} x}\left(\left(v_{N}^{\prime}-a_{x}\left(x, z_{N}\right)\right) f_{N}^{2}\right), \\
\frac{\mathrm{d}}{\mathrm{d} x} \hat{b}^{0}(x) & =\frac{r p \kappa^{2}}{2} f_{0} f_{1} \sin \Phi_{1,0}-D_{0}=-p \frac{\mathrm{d}}{\mathrm{d} x}\left(\left(v_{0}^{\prime}-a_{x}\left(x, z_{0}\right)\right) f_{0}^{2}\right) .
\end{aligned}
$$

In each case we integrate (and use the boundary conditions $\hat{b}^{n}(-L)=0=v_{n}^{\prime}(-L)-$ $\left.a_{x}\left(-L, z_{n}\right)\right)$ to verify that $\hat{b}^{n}$ satisfies conditions (38) and (39).

Next we define $\hat{b}(x, z)=\hat{b}^{n}(x)$ for $z_{n-1}<z<z_{n}$, and show that $\hat{b}$ also solves (41). By an integration by parts in each summand,

$$
\begin{aligned}
\sum_{n=1}^{N} & \int_{-L}^{L} \int_{z_{n-1}}^{z_{n}} \frac{1}{\kappa^{2}} \hat{b}(x, z) \Delta \eta \mathrm{d} z \mathrm{~d} x \\
= & \sum_{n=1}^{N} \int_{-L}^{L} \int_{z_{n-1}}^{z_{n}} \frac{1}{\kappa^{2}} \hat{b}^{n}(x) \Delta \eta \mathrm{d} z \mathrm{~d} x \\
= & \frac{1}{\kappa^{2}} \sum_{n=1}^{N}\left\{\int_{-L}^{L} \hat{b}^{n}(x)\left(\partial_{z} \eta\left(x, z_{n}\right)-\partial_{z} \eta\left(x, z_{n-1}\right)\right) \mathrm{d} x-\int_{-L z_{n-1}}^{L} \int_{z_{n}}^{z_{n}} \frac{\mathrm{d} \hat{b}^{n}}{\mathrm{~d} x} \partial_{x} \eta(x, z) \mathrm{d} z \mathrm{~d} x\right\} \\
= & \frac{1}{\kappa^{2}} \int_{-L}^{L}\left[\hat{b}^{N}(x) \partial_{z} \eta\left(x, z_{N}\right)-\hat{b}^{1}(x) \partial_{z} \eta(x, 0)\right] \mathrm{d} x \\
& +\frac{1}{\kappa^{2}} \sum_{n=1}^{N-1} \int_{-L}^{L}\left(\hat{b}^{n}(x)-\hat{b}^{n+1}(x)\right) \partial_{z} \eta\left(x, z_{n}\right) \mathrm{d} x \\
& -\sum_{n=1}^{N} \int_{-L}^{L} \int_{z_{n-1}}^{z_{n}}\left(\frac{r p}{2} f_{n} f_{n-1} \sin \Phi_{n, n-1}(x)-D_{n}\right) \partial_{x} \eta \mathrm{d} z \mathrm{~d} x
\end{aligned}
$$




$$
\begin{aligned}
= & p \sum_{n=0}^{N} \int_{-L}^{L} \frac{1}{\kappa^{2}}\left(v_{n}^{\prime}-a_{x}\left(x, z_{n}\right)\right) f_{n}^{2} \partial_{z} \eta\left(x, z_{n}\right) \mathrm{d} x \\
& -\sum_{n=1}^{N} \int_{-L}^{L} \int_{z_{n-1}}^{z_{n}} \frac{r p}{2} f_{n} f_{n-1} \sin \Phi_{n, n-1}(x) \partial_{x} \eta \mathrm{d} z \mathrm{~d} x
\end{aligned}
$$

for all $\eta \in H^{2} \cap H_{0}^{1}(D)$. That is, $\hat{b}$ also solves Eq. (41). Now consider the function $g(x, z)$ defined by $g(x, z)=b(x, z)-\hat{b}(x, z)$. Then $g \in L^{2}(D)$ and solves $\iint_{D} g(x, z) \Delta \eta \mathrm{d} x \mathrm{~d} z=0$ for all $\eta \in H^{2} \cap H_{0}^{1}(D)$. Taking $\eta$ to be the solution to the Dirichlet problem, $\Delta \eta=g,\left.\eta\right|_{\partial D}=0$ we arrive at the desired conclusion $g \equiv 0$, and hence $b(x, z)=\hat{b}(x, z)$ and the lemma is established.

As before, we observe that the gauge-invariant phase difference satisfies a useful equation (see (10)), which derives from Stokes' Theorem:

$$
\frac{\mathrm{d}}{\mathrm{d} x} \varphi_{n, n-1}(x)=\left(v_{n}^{\prime}-a_{x}\left(x, z_{n}\right)\right)-\left(v_{n-1}^{\prime}-a_{x}\left(x, z_{n-1}\right)\right)+p b^{(n)}(x) .
$$

We now begin the proof of Lemma 3.1.

Step 1. Getting started. By the implicit function theorem, applied to Eq. (20), for every $s \in \mathcal{S}$, and for every fixed choice of parameters $N, L, \kappa, H$, there exist constants $\rho>0, r_{1}>0$ such that (20) admits a unique solution $w=w(r, s)$ with $|r|<r_{1}$ and $\|w\|<\rho$. Since $\Omega_{r}(s+w(r, s))$ is periodic in $s$ we may compactify the problem by treating $\mathcal{S}$ as an $N$-torus, and hence the constants $r_{1}, \rho$ may be chosen independently of $s \in \mathcal{S}$. Statements (i)-(iii) of Lemma 3.1 then follow from standard arguments involving the Implicit Function Theorem and the regularity of the functional $\Omega_{r}$ (see [2]). What remains to prove is the dependence of the value $r_{1}$ on the various parameters.

We first observe that Lemma 3.1(i) implies that the solutions $w(r, s)$ satisfy a uniform (in $s$ ) estimate $\|w(r, s)\| \leqslant C_{1}|r|$, with constant $C_{1}$ possibly depending on all parameters, $N, L, \kappa, H, p$. From this preliminary argument we may, by reducing $r_{1}>0$ if necessary, assume that

$$
\frac{1}{2} \leqslant f_{n}(x) \leqslant \frac{3}{2}, \quad n=0, \ldots, N
$$

for $|r|<r_{1}$

Step 2. Uniform estimates in $n$. We now use the Euler-Lagrange equations (35)-(40) derived above to estimate the gauge-invariant quantities, in the supremum norm and individually in each plane or gap.

First we make rough bounds, then iterate to improve the dependence on parameters. Since we can estimate the constants $\left|C_{n}\right| \leqslant r$ and $\left|D_{n}\right| \leqslant r \kappa^{2} p / 2$, we have

$$
\begin{aligned}
\left\|b^{(n)}\right\|_{\infty} & \leqslant c r \kappa^{2} p L, \\
\left\|v_{n}^{\prime}-a_{x}\left(\cdot, z_{n}\right)\right\|_{\infty} & \leqslant c r \kappa^{2} L .
\end{aligned}
$$

(Here and in the following $c$ will denote a pure constant, independent of all other parameters.) 
Now consider the equations for $u_{n}$. Suppose first that $\max _{x, k} u_{k}(x)=u_{n}\left(x_{0}\right) \geqslant 0$. If $n \in\{1, \ldots, N-1\}$, we observe that the extremal property of $u_{n}\left(x_{0}\right)$ implies that

$$
u_{n-1}\left(x_{0}\right) \cos \Phi_{n, n-1}+u_{n+1}\left(x_{0}\right) \cos \Phi_{n+1, n}-2 u_{n}\left(x_{0}\right) \leqslant 0,
$$

while if $n=0$ or $n=N$ the corresponding terms

$$
u_{N-1}\left(x_{0}\right) \cos \Phi_{N, N-1}-u_{N}\left(x_{0}\right) \leqslant 0, \quad \text { or } \quad u_{1}\left(x_{0}\right) \cos \Phi_{1,0}-u_{0}\left(x_{0}\right) \leqslant 0 .
$$

Consequently, at the maximum point the right-hand side of (35) is non-positive. By the maximum principle we obtain $u_{n}\left(x_{0}\right)<0$. (Note this argument holds in general, for any value of the parameters, for any solution of the Lawrence-Doniach system.)

Next assume that $\min _{x, k} u_{k}(x)=u_{n}\left(x_{0}\right)<0$. By the same arguments as above at the point $x_{0}$, the extremal $u_{n}$ satisfies an equation of the form $-\frac{1}{\kappa^{2}} u_{n}^{\prime \prime}\left(x_{0}\right)+c\left(x_{0}\right) u_{n}\left(x_{0}\right) \geqslant$ $-\frac{1}{\kappa^{2}}\left(v_{n}^{\prime}-a_{x}\left(x_{0}, z_{n}\right)\right)^{2}-2 r$, with $c(x) \geqslant \frac{1}{2}$. In conclusion we may bound $u_{n}$ via

$$
0>u_{n}(x) \geqslant-\frac{2}{\kappa^{2}} \sup _{x}\left(v_{n}^{\prime}-a_{x}\left(x_{0}, z_{n}\right)\right)^{2}-4 r .
$$

Applying the simple estimate (45) we obtain a preliminary bound on $u_{n}, 0>u_{n}(x)>$ $-c r\left(r \kappa^{2} L^{2}+1\right)$. We use the calculus inequality

$$
\sup _{x \in[-L, L]}\left|f^{\prime}(x)\right| \leqslant \frac{2}{\delta} \sup _{x \in[-L, L]}|f(x)|+\frac{\delta}{2} \sup _{x \in[-L, L]}\left|f^{\prime \prime}(x)\right|, \quad \text { for any } 0<\delta \leqslant L,
$$

with $\delta=1$, and obtain $\left\|u_{n}^{\prime}\right\|_{\infty} \leqslant c r\left(r \kappa^{2} L^{2}+1\right)$.

To improve the above estimates we need to take into account the fact that the integrands and right-hand sides contain terms which oscillate rapidly when $H$ is large. In particular, $\Phi_{n, n-1}=H p x+\varphi_{n, n-1}$, and we expect that terms containing the sine or cosine of $\Phi_{n, n-1}$ tend to zero in the weak sense as $H \rightarrow \infty$. Since these oscillatory terms are sources in the equations for the fields and currents, we should obtain stronger convergence to zero as $H \rightarrow \infty$, and hence sharper estimates for large $H$ than we obtained in bluntly measuring these terms in the supremum norm. With this in mind, we require one further ingredient: a bound for $\varphi_{n, n-1}^{\prime}$ using Eq. (42),

$$
\sup _{x}\left|\varphi_{n, n-1}^{\prime}(x)\right| \leqslant c r \kappa^{2} L\left(1+p^{2}\right) .
$$

We now integrate by parts in $C_{n}, D_{n}$, and in the integrals representing $b^{(n)}(x)$ and $v_{n}^{\prime}-a_{x}\left(x, z_{n}\right)$ :

$$
\begin{aligned}
\sup _{x}\left|b^{(n)}(x)\right| & \leqslant c \frac{r \kappa^{2} p}{H p}\left(1+L\left\|u_{n}^{\prime}\right\|_{\infty}\right)+L\left\|\varphi_{n, n-1}^{\prime}\right\|_{\infty} \\
& \leqslant c \frac{r \kappa^{2} p}{H p}\left(1+r L^{2} \kappa^{2}\right)(1+r L),
\end{aligned}
$$


and similarly

$$
\sup _{x}\left|v_{n}^{\prime}(x)-a_{x}\left(x, z_{n}\right)\right| \leqslant c \frac{r \kappa^{2}}{H p}\left(1+r L^{2} \kappa^{2}\right)(1+r L) .
$$

Substituting into (46) we obtain a more refined estimate for $u_{n}$,

$$
0>u_{n}(x)>-C_{u} r\left(1+r \frac{\kappa^{2}}{H^{2} p^{2}}\left(1+r \kappa^{2} L^{2}\right)^{2}(1+r L)^{2}\right),
$$

with constant $C_{u}$ independent of $N, \kappa, L$.

Step 3. A lower bound on the linearization at $r=0$. We define

$$
\lambda=\lambda(N, L, \kappa, p):=\inf \left\{Q_{0}(\Psi, \Psi): \Psi=\left(\mu_{n}, v_{n}, \vec{\alpha}\right) \in W,\left\|\left(\mu_{n}, v_{n}, \vec{\alpha}\right)\right\|=1\right\},
$$

with

$$
\begin{array}{rl}
Q_{0}(\Psi, \Psi)= & D^{2} \Omega_{r=0}\left(f_{n}^{0}, \phi_{n}^{0}, \vec{A}^{0}\right)[\Psi, \Psi] \\
=p & p \sum_{n=0}^{N} \int_{-L}^{L}\left(\frac{1}{\kappa^{2}}\left(\mu_{n}^{\prime}\right)^{2}+2 \mu_{n}^{2}+\frac{1}{\kappa^{2}}\left(v_{n}^{\prime}-\alpha_{x}\left(x, z_{n}\right)^{2}\right)\right) \mathrm{d} x \\
& \quad+\frac{1}{\kappa^{2}} \int_{-L}^{L} \int_{0}^{N p}(\operatorname{curl} \alpha)^{2} \mathrm{~d} z \mathrm{~d} x .
\end{array}
$$

Recall that, as an operator on the whole tangent space $E, D^{2} \Omega_{0}(s)$ has an $N$-dimensional kernel for any $s \in \mathcal{S}$. Here we consider its restriction to the orthogonal subpace $W=$ $T \mathcal{S}^{\perp}=\operatorname{ker}\left(D^{2} \Omega_{0}(s)\right)^{\perp}$. From the proof of Proposition 2.1(b) we may conclude that $\lambda>0$ for any choice of $N, L, \kappa, p$. Here we will obtain the more precise information on its dependence on these parameters.

Note first that (trivially, since $\kappa \geqslant 1$ by hypothesis),

$$
p \sum_{n=0}^{N} \int_{-L}^{L}\left(\frac{1}{\kappa^{2}}\left(\mu_{n}^{\prime}\right)^{2}+\mu_{n}^{2}\right) \mathrm{d} x \geqslant \frac{1}{\kappa^{2}} p \sum_{n=0}^{N}\left\|\mu_{n}\right\|_{H^{1}}^{2} .
$$

We require the following lemma to estimate the terms including the vector potential $\vec{a}$ appearing in the linearizations:

LeMma 5.2. - For any $\vec{a} \in H^{1}(D)$ with $\vec{a} \cdot \hat{n}=0$ on $\partial D$,

$$
\begin{gathered}
p \sum_{n=0}^{N}\left\|a_{x}\left(\cdot, z_{n}\right)\right\|_{L^{2}(D)}^{2} \leqslant \frac{(p+1)(N+1)}{N}\left\|a_{x}\right\|_{H^{1}(D)}^{2}, \\
\sum_{n=1}^{N}\left\|\int_{z_{n-1}}^{z_{n}} a_{z}(z) \mathrm{d} z\right\|_{L^{2}(D)}^{2} \leqslant p \iint_{D} a_{z}^{2}(x, z) \mathrm{d} x \mathrm{~d} z .
\end{gathered}
$$


Proof. - Let $\chi_{n}^{+}(z)=\left(z-z_{n-1}\right) / p, n=1, \ldots, N$. Then we apply the divergence theorem to the vector field $\left(a_{x}^{2}(x, z) \chi_{n}^{+}, 0\right)$ in the strip $[-L, L] \times\left(z_{n-1}, z_{n}\right)$ to obtain

$$
\begin{aligned}
\int_{-L}^{L}\left(a_{x}^{2}\left(x, z_{n}\right)\right)^{2} \mathrm{~d} x & =\int_{-L z_{n-1}}^{L} \int_{\frac{z_{n}}{\partial}} \frac{\partial}{\partial z}\left(\chi_{n}^{+}(z) a_{x}^{2}(x, z)\right) \mathrm{d} z \mathrm{~d} x \\
& \leqslant\left[1+\frac{1}{p}\right] \int_{-L}^{L} \int_{z_{n-1}}^{z_{n}}\left(a_{x}^{2}+\left|\frac{\partial a_{x}}{\partial z}\right|^{2}\right) \mathrm{d} z \mathrm{~d} x
\end{aligned}
$$

for $n=1, \ldots, N$. Similarly, using $\chi_{n}^{-}=\left(z_{n+1}-z\right) / p$ in the strip $[-L, L] \times\left(z_{n}, z_{n+1}\right)$ we obtain

$$
\int_{-L}^{L}\left(a_{x}\left(x, z_{n}\right)\right)^{2} \mathrm{~d} x \leqslant\left[1+\frac{1}{p}\right] \int_{-L}^{L} \int_{z_{n}}^{z_{n+1}}\left(a_{x}^{2}+\left|\frac{\partial a_{x}}{\partial z}\right|^{2}\right) \mathrm{d} z \mathrm{~d} x,
$$

for $n=0, \ldots, N-1$.

Clearly there exists an index $n_{0}$ such that

$$
\int_{-L z_{n_{0}-1}}^{L} \int_{z_{0}}^{z_{n_{0}}}\left(a_{x}^{2}+\left|\frac{\partial a_{x}}{\partial z}\right|^{2}\right) \mathrm{d} z \mathrm{~d} x \leqslant \frac{1}{N} \iint_{D}\left(a_{x}^{2}+\left|\frac{\partial a_{x}}{\partial z}\right|^{2}\right) \mathrm{d} z \mathrm{~d} x .
$$

For $0 \leqslant n \leqslant n_{0}-1$ we use estimate (54) for $\left\|a_{x}\right\|_{2}^{2}$, and for $n_{0} \leqslant n \leqslant N$ we use (53). In doing so we require the $H^{1}$-norm of $a_{x}$ in each interval exactly once, except for the strip $z_{n_{0}-1}<z<z_{n_{0}}$ which appears twice. In this way we arrive at the desired estimate (51).

The estimate (52) is an elementary consequence of the Cauchy-Schwartz inequality.

We now estimate the second term of $Q_{0}$ : since

$$
2\left|v_{n}^{\prime} \alpha_{x}\left(x, z_{n}\right)\right| \leqslant(1-\varepsilon)\left(v_{n}^{\prime}\right)^{2}+\frac{1}{1-\varepsilon} \alpha_{x}^{2}\left(x, z_{n}\right),
$$

for any $\varepsilon>0$, we have

$$
\int_{-L}^{L}\left(v_{n}^{\prime}-\alpha_{x}\left(x, z_{n}\right)\right)^{2} \mathrm{~d} x \geqslant \int_{-L}^{L} \varepsilon\left(v_{n}^{\prime}\right)^{2} \mathrm{~d} x-\int_{-L}^{L} \frac{\varepsilon}{1-\varepsilon} \alpha_{x}^{2}\left(x, z_{n}\right) \mathrm{d} x .
$$

We now use (51) and the elliptic estimate (11)-(12) to estimate

$$
\begin{aligned}
& \iint(\operatorname{curl} \alpha)^{2} \mathrm{~d} x \mathrm{~d} z-p \sum_{n=0}^{N} \int_{-L}^{L} \frac{\varepsilon}{1-\varepsilon} \alpha_{x}^{2}\left(x, z_{n}\right) \mathrm{d} x \\
& \quad \geqslant\left(\frac{1}{2}\left(1+\frac{4}{\pi^{2}} \frac{L^{2} N^{2} p^{2}}{4 L^{2}+N^{2} p^{2}}\right)^{-2}-\frac{2 \varepsilon}{(1-\varepsilon)}\right)\|\vec{\alpha}\|_{H^{1}}^{2}
\end{aligned}
$$




$$
\geqslant\left(\frac{1}{2}\left(1+\frac{4}{\pi^{2}} L^{2}\right)^{-2}-\frac{2 \varepsilon}{(1-\varepsilon)}\right)\|\vec{\alpha}\|_{H^{1}}^{2},
$$

uniformly in $N$. Now choose $\varepsilon=\frac{1}{4} \min \left\{1, \frac{1}{2}\left(1+\frac{4}{\pi^{2}} L^{2}\right)^{-2}\right\}$. From the Poincaré inequality (for $H^{1}$ functions on $[-L, L]$ with vanishing mean) we may then obtain the lower bound

$$
\begin{aligned}
& p \sum_{n=0}^{N} \int_{-L}^{L}\left(v_{n}^{\prime}-\alpha_{x}\left(x, z_{n}\right)\right)^{2} \mathrm{~d} x+\iint_{D}(\operatorname{curl} \vec{\alpha})^{2} \mathrm{~d} x \mathrm{~d} z \\
& \geqslant \varepsilon p \sum_{n=0}^{N} \int_{-L}^{L}\left(v_{n}^{\prime}\right)^{2} \mathrm{~d} x+\frac{1}{4} \min \left\{1,\left(1+\frac{4}{\pi^{2}} L^{2}\right)^{-2}\right\}\|\vec{\alpha}\|_{H^{1}}^{2} \\
& \geqslant \varepsilon\left(1+\frac{4}{\pi^{2}} L^{2}\right)^{-1} p \sum_{n=0}^{N}\left\|v_{n}\right\|_{H^{1}}^{2}+\frac{1}{4} \min \left\{1,\left(1+\frac{4}{\pi^{2}} L^{2}\right)^{-2}\right\}\|\vec{\alpha}\|_{H^{1}}^{2} \\
& \geqslant \frac{1}{4} \min \left\{1,\left(1+\frac{4}{\pi^{2}} L^{2}\right)^{-3}\right\}\left[p \sum_{n=0}^{N}\left\|v_{n}\right\|_{H^{1}}^{2}+\|\vec{\alpha}\|_{H^{1}}^{2}\right] .
\end{aligned}
$$

We then obtain

$$
\lambda \geqslant \frac{1}{4 \kappa^{2}} \min \left\{1,\left(1+\frac{4}{\pi^{2}} L^{2}\right)^{-3}\right\} .
$$

Note in particular that $\lambda$ is bounded away from zero uniformly in $N$, but the bound deteriorates as either $L \rightarrow \infty$ or $\kappa \rightarrow \infty$. (See Remark 5.3.)

Step 4. An upper bound on the linearization. We define $Q_{r}$ to be the quadratic form representing the second variation of energy around the solution $s+w(r, s) \in \mathcal{S}_{r}$, with respect to variations $\Psi=\left(\mu_{n}, v_{n}, \vec{\alpha}\right)$ in the subspace $W$ :

$$
\begin{aligned}
Q_{r}(\Psi, \Psi):= & \left.\frac{1}{2} \frac{\mathrm{d}^{2}}{\mathrm{~d} t^{2}}\right|_{t=0} \Omega_{r}(s+w(r, s)+t \Psi) \\
= & p \sum_{n=0}^{N} \int_{-L}^{L}\left\{\frac{1}{\kappa^{2}}\left(\mu_{n}^{\prime}\right)^{2}+\frac{1}{\kappa^{2}}\left(v_{n}^{\prime}-a_{x}\right)^{2} \mu_{n}^{2}+\left(3 f_{n}^{2}-1\right) \mu_{n}^{2}\right. \\
& \left.+\frac{4}{\kappa^{2}}\left(v_{n}^{\prime}-a_{x}\right) f_{n} \mu_{n}\left(v_{n}^{\prime}-\alpha_{x}\left(x, z_{n}\right)\right)+\frac{1}{\kappa^{2}} f_{n}^{2}\left(v_{n}^{\prime}-\alpha_{x}\left(x, z_{n}\right)\right)^{2}\right\} \mathrm{d} x \\
& +\frac{r}{2} p \sum_{n=1}^{N} \int_{-L}^{L}\left\{\left(f_{n} \mu_{n-1}+f_{n-1} \mu_{n}\right) \sin \Phi_{n, n-1}\left[v_{n}-v_{n-1}-\int_{z_{n-1}}^{z_{n}} \alpha_{z} \mathrm{~d} z\right]\right. \\
& +f_{n} f_{n-1} \cos \Phi_{n, n-1}\left[v_{n}-v_{n-1}-\int_{z}^{z_{n}} \alpha_{z} \mathrm{~d} z\right]^{2} \\
& \left.+\mu_{n}^{2}+\mu_{n-1}^{2}-2 \mu_{n} \mu_{n-1} \cos \Phi_{n, n-1}\right\} \mathrm{d} x+\frac{1}{\kappa^{2}} \iint_{B}(\operatorname{curl} \vec{\alpha})^{2} \mathrm{~d} x \mathrm{~d} z .
\end{aligned}
$$


As long as the linearization $Q_{r}$ remains non-singular (as an operator on $W$ ), we may invoke the Implicit Function Theorem to ensure that the solutions to Eq. (20) in the form $s+w(r, s)$ determined by Lemma 3.1 remain valid. Hence, the critical value $r_{0}$ which determines the radius of convergence of the expansion is bounded below by the smallest value of $r$ for which $Q_{r}$ admits zero as an eigenvalue. If $Q_{r}$ degenerates at $r_{*}$, then there exists a test vector $\Psi \in W,\|\Psi\|=1$, such that $Q_{r_{*}}(\Psi, \Psi)=0$. Using the lower bound on $Q_{0}$, we have:

$$
\lambda \leqslant Q_{0}(\Psi, \Psi)=Q_{r_{*}}(\Psi, \Psi)+\left(Q_{0}-Q_{r_{*}}\right)(\Psi, \Psi)=\left(Q_{0}-Q_{r_{*}}\right)(\Psi, \Psi) .
$$

An estimate of the difference between the linearization at $r=r_{*}$ and at $r=0$ in terms of $r_{*}$ (and the norm of $\Psi$ ) will then yield a lower bound on the critical value $r_{0} \geqslant r_{*}$. This estimate follows from the sup-norm bounds on each $u_{n},\left(v_{n}^{\prime}-a_{x}\left(\cdot, z_{n}\right)\right)$ obtained in (50) and (49):

$$
\begin{aligned}
&\left|Q_{r_{*}}(\Psi, \Psi)-Q_{0}(\Psi, \Psi)\right| \\
& \leqslant p \sum_{n=0}^{N} \int_{-L}^{L}\left\{\frac{1}{\kappa^{2}}\left(v_{n}^{\prime}-a_{x}\right)^{2} \mu_{n}^{2}+3\left|f_{n}^{2}-1\right| \mu_{n}^{2}\right. \\
&\left.+\frac{4}{\kappa^{2}}\left|v_{n}^{\prime}-a_{x}\right|\left|\mu_{n}\right|\left|v_{n}^{\prime}-\alpha_{x}\left(x, z_{n}\right)\right|+\frac{1}{\kappa^{2}}\left|f_{n}^{2}-1\right|\left(v_{n}^{\prime}-\alpha_{x}\left(x, z_{n}\right)\right)^{2}\right\} \mathrm{d} x \\
&+\frac{r_{*}}{2} p \sum_{n=1}^{N} \int_{-L}^{L}\left\{\left(\left|\mu_{n-1}\right|+\left|\mu_{n}\right|\right)\left|v_{n}-v_{n-1}-\int_{z_{n-1}}^{z_{n}} \alpha_{z} \mathrm{~d} z\right|\right. \\
&\left.+\mu_{n}^{2}+\mu_{n-1}^{2}-2\left|\mu_{n} \mu_{n-1}\right|+\left[v_{n}-v_{n-1}-\int_{z_{n-1}}^{z_{n}} \alpha_{z} \mathrm{~d} z\right]^{2}\right\} \mathrm{d} x \\
& \leqslant c r_{*}\left[1+K\left(1+r_{*} \kappa^{2} K\right)\right]\|\Psi\|^{2},
\end{aligned}
$$

where $K:=\frac{1}{H p}\left(1+r_{*} L^{2} \kappa^{2}\right)\left(1+r_{*} L\right)$. Inserting the above estimate and (55) into (56) produces a lower bound on the first point of degeneracy $r_{*}$,

$$
r_{*}\left[1+K\left(1+r_{*} \kappa^{2} K\right)\right] \geqslant \lambda \geqslant \frac{1}{4 \kappa^{2}} \min \left\{1,\left(1+\frac{4}{\pi^{2}} L^{2}\right)^{-3}\right\} .
$$

We observe that the left-hand term is monotone increasing in $r_{*}$, and therefore implies a lower bound of the form $r_{0} \geqslant r_{*} \geqslant R(L, \kappa, H)$, where the constant $R$ can be chosen uniformly in $N$, but decreases with increasing $L$ and $\kappa$ and increases with increasing $H$.

Step 5. Conclusion. The above estimates have all been based on the (unsatisfactory) initial hypothesis $|r|<r_{1}(N, L, \kappa, H)$ chosen small enough to ensure (43). However, we observe that the only role of this hypothesis is to obtain (43), and the estimates obtained thereafter are valid whenever (43) holds. Since $\left\|u_{n}\right\|_{\infty}$ is a continuous function of $r$, the estimates obtained for $u_{n}, v_{n}, \vec{a}$ and $r_{*}$ above persist as we increase $r$, either until (43) is first violated or until we reach $r=r_{*}$. From (50), if condition (43) is violated then $r$ satisfies $C_{u} r\left[1+r \kappa^{2} K^{2}\right] \geqslant \frac{1}{2}$, a condition on $r$ which also defines a lower bound which 
is independent of $N$, decreasing with increasing $L, \kappa$, and increasing as $H$ increases (just as for $R(L, \kappa, H)$ above). In either case, the perturbation argument remains valid for an interval $r \in\left[0, r_{0}\right)$ with $r_{0}$ bounded below by a constant which is independent of $N$, with the dependences on $L, \kappa, H$ claimed in the statement of (iv). This concludes the proof of Theorem 3.1.

Remark 5.3. - We note that the lower bound on $r_{*}$ obtained above improves with smaller $L, \kappa$ and larger $H$. Of course this is only a one-sided bound, and we cannot be sure that the solutions obtained by the degenerate perturbation method really do cease to exist if $L$ or $\kappa$ are too large. Indeed, solutions with a similar form may persist beyond the range of validity of Lemma 3.1, and the estimates which gave the lower bound (57) are certainly not sharp. However, we can show that the smallest eigenvalue $\lambda(N, L, \kappa)$ of the linearization at $r=0$ does tend to zero as either $L, \kappa \rightarrow \infty$. From the Implicit Function Theorem we may then infer that $w(r, s)$ grows rapidly near $r=0$, which in turn suggests that the interval of validity is diminished as $L, \kappa$ increase.

LEMMA 5.4. - For any fixed $N$,

$$
\lambda(N, L, \kappa) \leqslant \frac{9}{2 \kappa^{2} p^{2} L^{2}} \rightarrow 0
$$

as $L, \kappa \rightarrow \infty$.

Proof. - As before, set $M=N p$. Let $\xi \in H^{2} \cap H_{0}^{1}(D)$ be the solution to the Dirichlet problem

$$
\Delta \xi=1,\left.\quad \xi\right|_{\partial D}=0,
$$

and define $\vec{\alpha}=\left(\partial_{z} \xi,-\partial_{x} \xi\right)$. Note that $\xi$ is even in $x$, and $\frac{1}{\kappa^{2}} \iint_{D}(\operatorname{curl} \vec{\alpha})^{2} \mathrm{~d} x \mathrm{~d} z=\frac{2 L M}{\kappa^{2}}$.

We next choose $v_{n}$ with $\frac{\mathrm{d}}{\mathrm{d} x} v_{n}(x)=\alpha_{x}\left(x, z_{n}\right), \int_{-L}^{L} v_{n}(x) \mathrm{d} x=0$. Since $\alpha_{x}(x, z)$ is even in $x$ we have $v_{n}(x)=\int_{0}^{x} \alpha_{x}\left(x^{\prime}, z_{n}\right) \mathrm{d} x^{\prime}$, and $v_{n}$ is odd. Applying Stokes' Theorem to the rectangle $[-L, L] \times\left[z_{n-1}, z_{n}\right]$,

$$
\begin{aligned}
2 p x & =\int_{z_{n-1}}^{z_{n}} \int_{-x}^{x} \operatorname{curl} \vec{\alpha} \mathrm{d} x \mathrm{~d} z \\
& =\int_{-x}^{x}\left(\alpha_{x}\left(x, z_{n}\right)-\alpha_{x}\left(x, z_{n-1}\right)\right) \mathrm{d} x+\int_{z_{n-1}}^{z_{n}}\left(\alpha_{z}(-x, z)-\alpha_{z}(x, z)\right) \mathrm{d} z \\
& =2\left(v_{n}(x)-v_{n-1}(x)-\int_{z_{n-1}}^{z_{n}} \alpha_{z}(x, z) \mathrm{d} z\right) .
\end{aligned}
$$

In particular,

$$
2 \sum_{n=0}^{N}\left\|v_{n}\right\|_{2}^{2} \geqslant \sum_{n=1}^{N}\left[\left\|v_{n}\right\|_{2}^{2}+\left\|v_{n-1}\right\|_{2}^{2}\right] \geqslant \frac{2 N p^{2}}{9} L^{3}-\sum_{n=1}^{N} \int_{-L}^{L}\left(\int_{z_{n-1}}^{z_{n}} \alpha_{z}(x, z) \mathrm{d} z\right)^{2} \mathrm{~d} x
$$




$$
\geqslant \frac{2 N p^{2}}{9} L^{3}-p \iint_{D}\left[\alpha_{z}(x, z)\right]^{2} \mathrm{~d} z \mathrm{~d} x
$$

Taking $\mu_{n}=0, \Psi=\left(\mu_{n}, v_{n}, \vec{\alpha}\right)$, we have

$$
\|\Psi\|_{E}^{2} \geqslant p \sum_{n=0}^{N}\left\|v_{n}\right\|_{2}^{2}+\|\vec{\alpha}\|_{2}^{2} \geqslant \frac{M p^{2}}{9} L^{3}
$$

assuming (as usual) that $0<p \leqslant 1$. We may then conclude that:

$$
\lambda(N, L, \kappa) \leqslant \frac{Q_{0}(\Psi, \Psi)}{\|\Psi\|_{E}^{2}} \leqslant \frac{\frac{1}{\kappa^{2}} \iint_{D}(\operatorname{curl} \vec{\alpha})^{2} \mathrm{~d} x \mathrm{~d} z}{\frac{M p^{2}}{9} L^{3}} \leqslant \frac{9}{2 \kappa^{2} p^{2} L^{2}} .
$$

\section{Acknowledgement}

The authors thank A. Bahri for his helpful suggestions for determining the region of validity of the perturbation methods. SA and LB wish to thank R.V. Kohn and P.A. Deift for inviting them to spend a semester at the Courant Institute in Fall 1999, and for the interest and encouragement they have shown throughout the years.

\section{REFERENCES}

[1] S. Alama, A.J. Berlinsky, L. Bronsard, Periodic vortex lattices for the Lawrence-Doniach model of layered superconductors in a parallel field, preprint, 2000, available on the preprint archive http://xxx.lanl.gov, math.AP/0010111.

[2] A. Ambrosetti, M. Badiale, Homoclinics: Poincaré-Melnikov type results via a variational approach, Ann. Inst. H. Poincaré Anal. Non Linéaire 15 (1998) 233-252.

[3] A. Ambrosetti, V. Coti-Zelati, I. Ekeland, Symmetry breaking in Hamiltonian systems, J. Differential Equations 67 (1987) 165-184.

[4] A. Bahri, Y. Li, O. Rey, On a variational problem with lack of compactness: the topological effect of the critical points at infinity, Calc. Var. Partial Differential Equations 3 (1995) 67-93.

[5] F. Bethuel, H. Brezis, F. Hélein, Ginzburg-Landau Vortices, Birkhauser, Boston, 1994.

[6] F. Bethuel, T. Riviére, Vortices for a variational problem related to superconductivity, Ann. Inst. H. Poincaré Anal. Non Linéaire 12 (1995) 243-303.

[7] L. Bulaevskii, Magnetic properties of layered superconductors with weak interaction between the layers, Sov. Phys. JETP 37 (1973) 1133-1136.

[8] L. Bulaevskii, J. Clem, Vortex lattice of highly anisotropic layered superconductors in strong, parallel magnetic fields, Phys. Rev. B44 (1991) 10234-10238.

[9] S. Chapman, Q. Du, M. Gunzburger, On the Lawrence-Doniach and anisotropic GinzburgLandau models for layered superconductors, SIAM J. Appl. Math. 55 (1995) 156-174.

[10] J. Clem, M. Coffey, Viscous flux motion in a Josephson-coupled layer model of high- $T_{c}$ superconductors, Phys. Rev. B42 (1990) 6209-6216.

[11] M. Del Pino, P. Felmer, Local minimizers for the Ginzburg-Landau energy, Math. Z. 225 (1997) 671-684. 
[12] T. Giorgi, D. Phillips, The breakdown of superconductivity due to strong fields for the Ginzburg-Landau model, SIAM J. Math. Anal. 30 (1999) 341-359.

[13] P. Grisvard, Elliptic Problems in Nonsmooth Domains, Pitman Advanced Publishing Program, Boston, 1985.

[14] C. Gui, Multipeak solutions for a semilinear Neumann problem, Duke Math. J. 84 (1996) 739-769.

[15] Y. Iye, How anisotropic are the cuprate high $T_{c}$ superconductors?, Comments Cond. Mat. Phys. 16 (1992) 89-111.

[16] P. Kes, J. Aarts, V. Vinokur, C. van der Beek, Dissipation in highly anisotropic superconductors, Phys. Rev. Lett. 64 (1990) 1063-1066.

[17] S. Kuplevakhsky, Microscopic theory of weakly couple superconducting multilayers in an external magnetic field, preprint cond-mat/9812277.

[18] W. Lawrence, S. Doniach, Proceedings of the Twelfth International Conference on Low Temperature Physics, E. Kanda (Ed.), Academic Press of Japan, Kyoto, 1971, p. 361.

[19] Y. Li, L. Nirenberg, The Dirichlet problem for singularly perturbed elliptic equations, Comm. Pure Appl. Math. 51 (1998) 1445-1490.

[20] E. Lieb, M. Loss, Analysis Graduate Studies in Mathematics, Vol. 14, American Mathematical Society, Providence, RI, 1997.

[21] J. Rubinstein, M. Schatzman, Asymptotics for thin superconducting rings, J. Math. Pures Appl., série 977 (1998) 801-820.

[22] O. Rey, Blow-up points of solutions to elliptic equations with limiting nonlinearity, Differential Integral Equations 4 (1991) 1155-1167.

[23] S. Theorodakis, Theory of vortices in weakly-Josephson-coupled layered superconductors, Phys. Rev. B42 (1990) 10172-10177.

[24] M. Tinkham, Introduction to Superconductivity, 2nd edn., Mc Graw-Hill, New York, 1996.

[25] J. Wei, On the interior spike solutions for some singular perturbation problems, Proc. Roy. Soc. Edinburgh, Sect. A 128 (1998) 849-874. 